\title{
Impact Assessment of the L-Band Digital Aeronautical Communications System on the Joint Tactical Information Distribution System
}

\author{
Miguel A. Bellido-Manganell, Thomas Gräupl and Michael Schnell
}

\begin{abstract}
The L-band digital aeronautical communications system (LDACS) is the future air-ground communications technology currently undergoing the International Civil Aviation Organization (ICAO) standardization process. As LDACS is intended to operate in the frequency band 960-1164 MHz, compatibility tests between LDACS and the legacy systems operating in this frequency band are necessary to ensure that no system is harmfully interfered. One of these systems is the joint tactical information distribution system (JTIDS), a technology employed by the tactical data link Link 16. In this paper, we present the results of an impact assessment of LDACS on JTIDS conducted through simulations. The extent of the impact has been quantified by simulating a wide variety of interference configurations, which are expected to cover most realistic interference conditions between LDACS and JTIDS. Baseband simulation models of both systems have been implemented and an interference scenario between LDACS and JTIDS has been defined. To evaluate the impact, the degradation of the signal-to-noise ratio (SNR) of JTIDS by the presence of LDACS has been determined. Default JTIDS transmissions, where the information is repeated at distant frequencies, do not show a significant degradation by the presence of strong LDACS interference, with an SNR loss lower than $1 \mathrm{~dB}$ in any considered interference scenario. Comparatively, a certain dependency on the specific LDACS deployment is noticeable for less protected JTIDS transmissions. Based on the observed interdependencies, recommendations for the deployment of LDACS are given in this paper, such that the impact of LDACS on JTIDS is minimized.
\end{abstract}

Index Terms-Future communications infrastructure (FCI), interference, L-band digital aeronautical communications system (LDACS), Link 16, multifunctional information distribution system (MIDS), tactical data link (TDL), joint tactical information distribution system (JTIDS).

\section{INTRODUCTION}

$\mathbf{F}$ LYING has become an indispensable way to travel and transport cargo in the last decades. According to [1], 35\% of world trade by value is carried by aircraft and around 3.5 billion passengers used air transport in 2015. Additionally, flight forecasts indicate that the number of flights will continuously grow in the following years, e.g., the number of instrumental flight rules (IFR) flight movements over Europe is expected to increase by $14.04 \%$ from 2016 to 2023 [2]. The

Copyright (c) 2015 IEEE. Personal use of this material is permitted. However, permission to use this material for any other purposes must be obtained from the IEEE by sending a request to pubs-permissions@ieee.org.

M. A. Bellido-Manganell, T. Gräupl, and M. Schnell are with the Institute of Communications and Navigation, German Aerospace Center (DLR), Münchener Straße 20, 82234 Weßling, Germany (email: Miguel.BellidoManganell@DLR.de; Thomas.Graeupl@DLR.de; Michael.Schnell@DLR.de). increasing number of flights will lead to a denser airspace and a more challenging air traffic management (ATM) in the near future.

Currently, data communications needed for ATM are supported by the aeronautical mobile (route) service $(\mathrm{AM}(\mathrm{R}) \mathrm{S})$ in the frequency band 117.975-137 MHz. However, this band is reaching saturation in some regions of the world and it will not be sufficient to cover the data communications requirements foreseen in the medium and long term [3], [4]. Consequently, the World Radiocommunication Conference (WRC) allocated the frequencies $960-1164 \mathrm{MHz}$ in the L-band for the operation of $\mathrm{AM}(\mathrm{R}) \mathrm{S}$ systems, under the condition that they shall not cause harmful interference to any aeronautical radionavigation system (ARNS) operating in this band [4].

For the ATM modernization, large-scale research and development projects have been launched, e.g., Single European Sky ATM Research [5] in Europe and Next Generation Air Transportation System [6] in the United States. Both projects aim to modernize the ATM by introducing new services and operational concepts, which shall be enabled by modern communications, navigation, and surveillance technologies. To this end, communication capabilities during all phases of flight shall be supported by a set of data link technologies integrated into a single communications network, which is known as the future communications infrastructure (FCI). The air-ground segment of the FCI will be covered by the L-band digital aeronautical communications system (LDACS). Two proposal for LDACS were initially developed: LDACS1 [7] and LDACS2 [8]. However, only LDACS1 is being currently considered in the undergoing LDACS standardization process of the International Civil Aviation Organization (ICAO) and, therefore, we restrict our analysis to LDACS1, hereinafter referred to as LDACS.

LDACS supports data and voice communications between ground stations (GSs) and aircraft equipped with an airborne station (AS). By employing frequency-division duplexing, LDACS supports simultaneous transmissions from the GS in the forward link (FL) and from the AS in the reverse link (RL). In both FL and RL, LDACS uses orthogonal frequencydivision multiplexing (OFDM) to separately modulate up to 50 OFDM sub-carriers spaced $9.765625 \mathrm{kHz}$ apart, leading to an occupied bandwidth of $495.05 \mathrm{kHz}$ per FL or RL channel. Moreover, LDACS employs adaptive coding and modulation, achieving data rates of up to $2.6 \mathrm{Mbps}$ for a pair of FL and RL channels [9].

Following the WRC frequency allocation for AM(R)S sys- 
tems, LDACS has been designed to operate in the L-band between 960 and $1164 \mathrm{MHz}$. However, since LDACS is only allowed to operate under the condition that no ARNS is harmfully affected, compatibility tests between LDACS and the legacy systems of the L-band are necessary.

The main user of the L-band is the distance measuring equipment (DME), a pulsed system used to measure the slant range between an airborne interrogator and a ground transponder. As DME operates in the frequency range 962$1213 \mathrm{MHz}$, covering practically the entire band where LDACS may operate, compatibility tests between LDACS and DME were considered crucial. In [10], the impact of LDACS on DME was measured and conditions for the operation of LDACS were extracted, under which the functionality of DME is not harmfully affected. Conversely, the impact of DME on LDACS was analysed in [11] and effective interference mitigation schemes for LDACS receivers have been proposed, e.g., in [12], [13].

Other systems operating in the L-band, such as the universal access transceiver (UAT) and the secondary surveillance radar (SSR), use only a small part of the L-band for their operation, i.e. UAT operates at $978 \mathrm{MHz}$ and SSR at $1030 \mathrm{MHz}$ and 1090 $\mathrm{MHz}$. Therefore, LDACS can minimize mutual interference with these systems with a certain frequency separation and sufficiently stringent frequency filters.

Another user of the L-band is Link 16, a tactical data link used by the military forces of the North Atlantic Treaty Organization (NATO) and some other countries [14]. Link 16 employs joint tactical information distribution system (JTIDS) and multifunctional information distribution system (MIDS) terminals to exchange real time tactical data among units [14]. Since this paper focuses on the communications component of Link 16, i.e., JTIDS or MIDS, and MIDS is a technology program intended to reduce the size and weight of the Link 16 terminals whilst maintaining JTIDS functionality [15], we do not make any difference between Link 16, JTIDS, and MIDS for the purpose of our analysis.

JTIDS implements frequency hopping among 51 frequency channels distributed between 969 and $1206 \mathrm{MHz}$ [14]-[16]. Since the JTIDS operational band covers almost the entire band intended for LDACS operation, mutual interference between LDACS and JTIDS is expected. The impact of JTIDS on LDACS was evaluated in [11], where simulations showed that LDACS can cope with JTIDS interference by implementing a simple interference mitigation technique, i.e., pulse blanking. However, a thorough analysis of the impact of LDACS on JTIDS has not yet been conducted.

In this paper, we present an impact assessment of LDACS on JTIDS. The impact of LDACS on JTIDS has been measured by comparing the performance of JTIDS obtained without LDACS interference, with its performance obtained under LDACS interference. Simulation models of both systems have been implemented and an interference scenario has been defined. The interference scenario has been simulated using a wide variety of interference configurations, which are expected to cover most realistic interference conditions that may arise between both systems in the future.

The remainder of this paper is organized as follows. The implemented LDACS and JTIDS models are described in Section II and Section III, respectively. The interference scenario and the different interference configurations are discussed in Section IV. In Section V, simulation results are presented and analysed. The conclusions of our analysis are given in Section VI.

\section{L-BAnd Digital Aeronautical Communications SYSTEM}

In order to simulate the interference of LDACS on JTIDS, baseband models of GS and AS transmitters have been implemented according to the publicly available LDACS specification [7]. Therefore, only the relevant parameters for our analysis are discussed in this section.

LDACS is a cellular point-to-multipoint communications system, where each GS serves ASs located within a certain volume of space called cell. The GS controlling each cell transmits continuously an OFDM-modulated signal using the FL frequency channel assigned to the cell. Its transmissions are structured in frames, which can be either broadcast or data/common control frames. Without loss of generality, the implemented GS transmits only data/common control frames. Each frame consists of 54 OFDM symbols, each one with a duration of $120 \mu \mathrm{s}$ and spanning 64 sub-carriers.

Transmissions from the ASs registered to each cell are organized by the GS following a combination of orthogonal frequency-division multiple access (OFDMA) and timedivision multiple access (TDMA). The RL is structured in super-frames of $240 \mathrm{~ms}$, each one consisting of an initial random access period of $6.72 \mathrm{~ms}$ and four multi-frames of $58.32 \mathrm{~ms}$ each. For simplicity, we neglect the random access period and assume that the RL is only composed of multiframes. Each multi-frame is subdivided into tiles of $0.72 \mathrm{~ms}$ composed of 6 OFDM symbols each. Each tile spans only the sub-carriers located either above or below the carrier frequency, with the exception of the first tile of each multiframe, i.e., the synchronization tile, which spans all subcarriers. Even though each tile could be assigned to a different AS, we simplify our analysis by assuming that each block of two simultaneous tiles, hereinafter referred to as a full-tile, is always assigned to one AS.

GSs and ASs process the data to be transmitted in a similar way. Data bits, which are generated randomly in our implementation, are randomized, encoded, and interleaved using the default encoding scheme defined in [7]. The resulting data bits are mapped to the data symbols of the constellation defined for the used modulation scheme, which can be either quadrature phase-shift keying (QPSK), 16-quadrature amplitude modulation (16-QAM), or 64-QAM. In our analysis, unless mentioned explicitly, we consider the default modulation scheme, QPSK. The obtained data symbols are mapped to their corresponding sub-carriers of each OFDM symbol. Other sub-carriers not carrying data symbols are modulated by using either synchronization symbols, pilot symbols, peakto-average-power-reduction symbols, or null symbols. After modulating all sub-carrier of each OFDM symbol, the inverse Fourier transform is applied to the OFDM symbol to obtain 
the time domain waveform. A cyclic prefix and a cyclic postfix are first added respectively at the beginning and at the end of each time domain OFDM symbol and then multiplied with the windowing function specified in [7]. Finally, the complex baseband signal is obtained by partially overlapping the cyclic prefixes and postfixes of consecutive OFDM symbols.

The complex baseband signal, generated by either a GS or an AS, is used as baseband interference in our simulations, as described in Section IV.

\section{LINK 16 - JOINT TACTICAL INFORMATION DisTRIBUTION SYSTEM}

Unlike the LDACS specification, the official specification of JTIDS is classified. Due to this, we have implemented both a JTIDS-type transmitter and a JTIDS-type receiver based on publicly available literature, such as guidebooks [14], instructions [15], reports [16], [17], and dissertations [18]. In case of discrepancies between the different sources, the most consistent or reliable parameters have been considered for our implementation.

JTIDS employs TDMA to organize transmissions from different JTIDS units (JUs) in time slots of $7.8125 \mathrm{~ms}$ each [14], [16]. The access to the time slots by the participating JUs can be either dedicated or shared with other JUs [14], [16]. We only consider dedicated access in our analysis.

Synchronization within the Link 16 network is achieved by designating a network time reference (NTR), which periodically broadcasts network entry messages used by the participating JUs to achieve coarse synchronization [14]. Fine synchronization is achieved either by exchanging round-triptiming (RTT) messages with the NTR, or by listening to special messages exchanged by the network and using a navigation system [14]. Additionally, JTIDS transmissions within a time slot include specific pulses for synchronization and time refinement. Synchronization process can also be simplified if the Global Positioning System (GPS) is available [14].

Given that specific messages can be exchanged to achieve fine synchronization, and that a terminal in fine synchronization can operate for up to three hours with sufficient time accuracy [14], we assume in our analysis that the members of the Link 16 network are correctly synchronized despite LDACS interference.

JTIDS transmissions are composed of pulses. Each pulse has an active part of $6.4 \mu \mathrm{s}$, where the information is transmitted, followed by an inactive part of $6.6 \mu \mathrm{s}$ [14], [16]. After the inactive part, another pulse is transmitted, leading to a pulse period of $13 \mu \mathrm{s}$ [14], [16]. Information can be carried either by one pulse, known as single-pulse, or transmitted twice in two consecutive pulses, known as double-pulse [14], [16]. Depending on the message structure, a JU can transmit 72, 258 , or 444 pulses during one time slot [15]-[17].

JTIDS uses frequency hopping in the Mode 1 of operation, which is the normal operational mode required by the current spectrum certification [14]. The 51 carrier frequencies used by JTIDS for frequency hopping, $\boldsymbol{f}^{\mathrm{J}}=\left(f_{0}^{\mathrm{J}}, f_{1}^{\mathrm{J}}, \ldots, f_{50}^{\mathrm{J}}\right)^{\mathrm{T}}$, are distributed uniformly every $3 \mathrm{MHz}$ in three sub-bands: 969$1008 \mathrm{MHz}, 1053-1065 \mathrm{MHz}$, and 1113-1206 MHz [14]-[17].
Consecutive pulses are transmitted in different frequencies [14], [16] with a minimum frequency separation of $30 \mathrm{MHz}$ [19]. Therefore, information transmitted using double-pulses is transmitted repeatedly in two distant frequencies. The frequency hopping pattern is pseudorandom and it must ensure that all frequency channels are used uniformly [16].

A Link 16 network can be composed of different subnetworks, which can operate concurrently in the same geographical area by using exclusive frequency hopping patterns, such that transmissions from different subnetworks do not coincide in time and frequency [16]. Although, theoretically, up to 127 subnetworks can operate using different frequency hopping patterns [14], [16], only up to 20-30 can coexist without significant mutual interference and, typically, no more than 3 to 6 subnetworks operate at the same time [14].

Even though JTIDS uses frequency hopping in its normal mode of operation [14], it can also operate at $969 \mathrm{MHz}$ without using frequency hopping [16] prior to the approval of a frequency assignment waiver [14]. Given that the LDACS $\mathrm{RL}$ is expected to operate using frequencies around $969 \mathrm{MHz}$ [20], JTIDS operation without frequency hopping might be affected by LDACS transmissions in the RL. However, as it is not the normal mode of operation of JTIDS and no information regarding its real usage has been disclosed, we do not consider the single-channel operation of JTIDS in our analysis. Nevertheless, we recommend a specific analysis on the single-channel operation of JTIDS and, if necessary, to conduct specific compatibility tests.

During its assigned time slot, a JU can transmit either data or an RTT message. Since we have assumed that all JUs are perfectly synchronized, only data transmissions are considered in our analysis. Four different message structures can be used to transmit data within a time slot [15], [16]:

- Standard double-pulse (STD) message structure: At the beginning of the time slot, nothing is transmitted into the channel during a variable pseudorandom delay of up to $2.418 \mathrm{~ms}$ known as jitter. After the jitter, 16 doublepulses for synchronization are transmitted, followed by 4 double-pulses for time refinement, 16 double-pulses carrying the header, and 93 double-pulses carrying data. The remaining part of the time slot is left as a propagation guard. The remaining formats described below are based on the STD message structure.

- Packed-2 single-pulse (P2SP) message structure: In this case, 186 single-pulses are available for data instead of 93 double-pulses.

- Packed-2 double-pulse (P2DP) message structure: Jitter is removed and 186 double-pulses are available for data.

- Packed-4 single-pulse (P4SP) message structure: Jitter is removed and 372 single-pulses are available for data.

Note that synchronization information, time refinement information, as well as the header, are always transmitted in double-pulses. Data are transmitted in double-pulses when using STD (by default) and P2DP messages, and in singlepulses when using P2SP and P4SP messages.

Different types of data can be transmitted by JTIDS [14]. Link 16 defines a set of predefined commands known as $\mathrm{J}$-series messages. Each $\mathrm{J}$-series message is composed of 
a different amount of predefined blocks of 70 bits, known as fixed-format (FF) words. Additionally, Link 16 supports the transmission of variable format (VF) words of 70 bits each, used for the definition of commands or messages not included in the specification. The transmission of free text (FT) messages of 75 bits each is also considered by Link 16 . FT messages can be used to carry voice, supporting $2.4 \mathrm{kbps}$ encoded or unencoded voice, and $16 \mathrm{kbps}$ unencoded voice. The type of data transmitted during a time slot is indicated in the header, which has a fixed length of 35 bits.

Before encoding the data, the 70-bit words (either FF or VF) are packed into blocks of 3 words (210 bits). Using each 3 -word block and 15 bits of the header, 12 bits of parity are obtained by employing a cyclic redundancy check (CRC) algorithm [18], [21]. A block of 225 bits is formed with the original 3-word block, the resulting 12 parity bits, and 3 spare bits [18], [21]. No parity bits are added to the FT messages [14], which can directly form blocks of 225 bits with 3 FT messages per block.

The 225-bit blocks are then encrypted and the encryption parameters are stored in the header [18], [21]. After the encryption, each 225-bit block is separated again in 3 words of 75 bits each.

Since both the CRC $(237,225)$ algorithm and the encryption parameters have not been disclosed, we assume in our implementation that both the encrypted data and the header can be approximated by pseudorandom uniform binary sequences.

\section{A. JTIDS-type Transmitter}

The implemented JTIDS-type transmitter, shown in Fig. 1, generates the data to be transmitted during a time slot as a pseudorandom uniform binary matrix $\boldsymbol{B}=\left[\begin{array}{llll}\boldsymbol{b}_{0}^{\mathrm{d}} & \boldsymbol{b}_{1}^{\mathrm{d}} & \ldots & \boldsymbol{b}_{L-1}^{\mathrm{d}}\end{array}\right]$, where $\boldsymbol{b}_{l}^{\mathrm{d}} \in\{0,1\}^{75}$ represents the $l^{\text {th }}$ data word of 75 bits. The number of words transmitted during a time slot, $L$, depends on the message structure: $L=3$ for STD, $L=6$ for P2SP and P2DP, and $L=12$ for P4SP. The header is also generated as a pseudorandom uniform binary vector $\boldsymbol{b}^{\mathrm{h}}$ of 35 bits, i.e., $\boldsymbol{b}^{\mathrm{h}} \in\{0,1\}^{35}$.

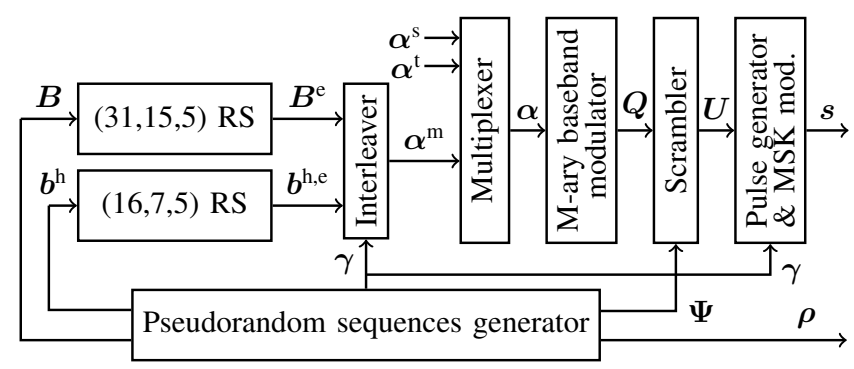

Fig. 1. Implemented JTIDS-type transmitter.

1) Encoding: The header $\boldsymbol{b}^{\mathrm{h}}$ is encoded onto a vector $\boldsymbol{b}^{\mathrm{h}, \mathrm{e}}$ of 16 5-bit symbols, hereinafter referred to as RS-symbols, using a $(16,7,5)$ Reed-Solomon (RS) encoding scheme [14].

Data may or may not be encoded. Whilst FF and VF are always RS encoded, FT messages may or may not be RS encoded [14]. Each FF and VF word is encoded separately onto $31 \mathrm{RS}$-symbols using a $(31,15,5) \mathrm{RS}$ encoding scheme
[14]. When FT messages are encoded, each block of 225 bits is RS encoded onto 465 bits [14]. Since the encoding rate is the same for any kind of encoded data, we consider in our analysis that data is always encoded using the same (31, $15,5) \mathrm{RS}$ encoder. Therefore, each data word $b_{l}^{\mathrm{d}}$, for $l=$ $0,1, \ldots, L-1$, is encoded separately onto an encoded data word $\boldsymbol{b}_{l}^{\mathrm{d}, \mathrm{e}}$ of $31 \mathrm{RS}$-symbols using a $(31,15,5) \mathrm{RS}$ encoding scheme [14]. The $L$ encoded data words form the matrix $\boldsymbol{B}^{\mathrm{e}}=$ $\left[\begin{array}{llll}\boldsymbol{b}_{0}^{\mathrm{d}, \mathrm{e}} & \boldsymbol{b}_{1}^{\mathrm{d}, \mathrm{e}} & \ldots & \boldsymbol{b}_{L-1}^{\mathrm{d}, \mathrm{e}}\end{array}\right]$.

In our implementation, only the transmission of encoded data has been considered, since it is assumed to be the most common case. Consequently, we assume our analysis to be valid for all types of data except for non-encoded FT messages.

2) Interleaving: After the encoding, the RS-symbols of header and data are interleaved together at RS-symbol level [14], forming a vector $\boldsymbol{\alpha}^{\mathrm{m}}$ of $16+31 \cdot L$ RS-symbols. The interleaving pattern $\gamma$ is assumed to be pseudorandom.

3) Multiplexing: 16 5-bit symbols $\boldsymbol{\alpha}^{\mathrm{s}}$ are appended before the message for receiver synchronization, followed by 45 bit symbols $\boldsymbol{\alpha}^{\mathrm{t}}$ for time refinement. For simplicity, all 5-bit symbols are further referred to as RS-symbol. The output of this block is a vector $\boldsymbol{\alpha}=\left[\boldsymbol{\alpha}^{\mathrm{s}} \boldsymbol{\alpha}^{\mathrm{t}} \boldsymbol{\alpha}^{\mathrm{m}}\right]$ composed of $A=$ $36+31 \cdot L$ RS-symbols.

4) M-ary Baseband Modulation: Each RS-symbol $\alpha_{a}$, for $a=0,1, \ldots, A-1$, is mapped onto a pattern $\boldsymbol{p}_{h}$ of 32 chips depending on its value. ${ }^{1}$ Since a RS-symbol can take up to 32 different values, 32 different patterns are used, i.e., $h=0,1, \ldots, 31$. The set $\boldsymbol{P}=\left[\begin{array}{llll}\boldsymbol{p}_{0} & \boldsymbol{p}_{1} \ldots \boldsymbol{p}_{31}\end{array}\right]$ of patterns used by JTIDS is obtained by cyclically shifting an initial pattern $\boldsymbol{p}_{0}$ [16], [18]. According to [18], $\boldsymbol{p}_{0}=(01111100111010010000101011101100)^{\mathrm{T}}$ and the cyclic shifting is done from right to left, i.e., $\boldsymbol{p}_{h}=$ $\left(p_{h-1,1}, p_{h-1,2}, \ldots, p_{h-1,31}, p_{h-1,0}\right)^{\mathrm{T}}$ for $h=1,2, \ldots, 31$. The output of this block for a RS-symbol $\alpha_{a}$ with a value $h$ is $\boldsymbol{q}_{a}=\boldsymbol{p}_{h}$. For a complete time slot, the output of this block is $\boldsymbol{Q}=\left[\begin{array}{llll}\boldsymbol{q}_{0} & \boldsymbol{q}_{1} & \ldots & \boldsymbol{q}_{A-1}\end{array}\right]$.

5) Scrambling: Each pattern $\boldsymbol{q}_{a}$ is mixed with a 32-chip sequence of pseudorandom noise $\boldsymbol{\psi}_{a} \in\{0,1\}^{32}$ using an exclusive-or (XOR) logical operator [18], such that $\boldsymbol{U}=\boldsymbol{Q} \oplus$ $\boldsymbol{\Psi}$, where $\boldsymbol{\Psi}=\left[\begin{array}{llll}\boldsymbol{\psi}_{0} & \boldsymbol{\psi}_{1} & \ldots & \boldsymbol{\psi}_{A-1}\end{array}\right], \boldsymbol{U}=\left[\begin{array}{llll}\boldsymbol{u}_{0} & \boldsymbol{u}_{1} & \ldots & \boldsymbol{u}_{A-1}\end{array}\right]$, and $\boldsymbol{u}_{a} \in\{0,1\}^{32}$.

6) Minimum-shift Keying Modulation: Each scrambled sequence $\boldsymbol{u}_{a}$ is transmitted in either a single-pulse or a doublepulse. From the initial $A$ sequences, $V$ pulses are transmitted during a time slot: $V=258$ pulses for STD and P2SP, and $V=444$ pulses for P2DP and P4SP. Pulses are modulated using minimum-shift keying (MSK) modulation [16], [18], with a chip period of $0.2 \mu$ s [14], [18].

The JTIDS-type transmitter implements the MSK modulation described in [22] without considering the up-conversion part. For each time slot, a complex baseband signal $s=$ $\left(s_{0}, s_{1}, \ldots, s_{K-1}\right)^{\mathrm{T}}$ is generated, where $K=\frac{13 \mu \mathrm{s}}{0.2 \mu \mathrm{s}} \cdot V$ is the number of samples comprising $s$.

In a real JTIDS transmitter, pulses are modulated, filtered, and up-converted to their corresponding frequencies according

\footnotetext{
${ }^{1}$ This process is also commonly referred to as cyclic code shift keying modulation in the literature (e.g. in [14], [16], [18]).
} 
to the frequency hopping pattern [16]. The measured spectrum of a transmitted pulse has an approximate $3 \mathrm{~dB}$ bandwidth of $3 \mathrm{MHz}$ [16], [23] and the transmission power is limited during peacetime to $200 \mathrm{~W}$ [15], [16].

In our analysis, we have considered a baseband implementation of JTIDS. Thus, the implemented JTIDS-type transmitter outputs the complex baseband signal $s$ and the generated frequency hopping pattern $\rho$, which indicates the frequency $\rho_{k} \in \boldsymbol{f}^{\mathrm{J}}$ where each complex sample $s_{k}$ is transmitted, for $k=0,1, \ldots, K-1$.

\section{B. JTIDS-type Receiver}

JTIDS terminals are periodically loaded with cryptographic keys [14]. These keys are used, together with the time slot identifier and the Link 16 subnetwork number, to perform the encryption, interleaving, scrambling, and frequency hopping [14]. For an unauthorized viewer without the correct keys, these processes seem to be random, whilst any authorized JTIDS receiver is perfectly capable of undoing them.

Since the frequency hopping pattern $\rho$ is known by the receiver and we consider a baseband model of the JTIDS receiver, we assume that the JTIDS receiver is perfectly capable of filtering, down-converting, and sampling the pulses transmitted in its subnetwork. Therefore, our implemented JTIDS-type receiver, shown in Fig. 2, receives directly the complex baseband signal $\boldsymbol{r}=\left(r_{0}, r_{1}, \ldots, r_{K-1}\right)^{\mathrm{T}}$.

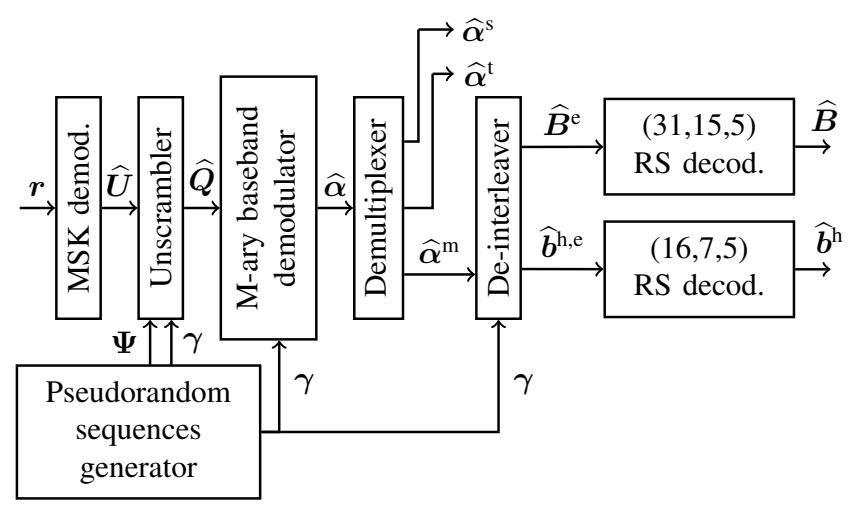

Fig. 2. Implemented JTIDS-type receiver.

1) MSK Demodulation: The implemented JTIDS-type receiver demodulates the received signal $\boldsymbol{r}$ by using the coherent MSK demodulation technique described in [22] without considering the down-conversion part. This technique has also been used in other implementations of a JTIDS-type receiver, e.g., in [18]. By demodulating each received pulse, a sequence of 32 chips $\widehat{\boldsymbol{u}}_{v}$ is obtained, where $v=0,1, \ldots, L-1$. Note that the samples corresponding to the inactive part of the pulses are discarded by the receiver. Given that $V$ pulses are received during a time slot, the output of this block is $\widehat{\boldsymbol{U}}=\left[\begin{array}{llll}\widehat{\boldsymbol{u}}_{0} & \widehat{\boldsymbol{u}}_{1} & \ldots & \widehat{\boldsymbol{u}}_{V-1}\end{array}\right]$.

2) Unscrambling: Each sequence $\widehat{\boldsymbol{u}}_{v}$ is unscrambled using an XOR operation with the same pseudorandom sequence $\boldsymbol{\psi}_{a}$ used by the JTIDS-type transmitter to scramble it, i.e., $\widehat{\boldsymbol{q}}_{v}=$ $\widehat{\boldsymbol{u}}_{v} \oplus \boldsymbol{\psi}_{a}$ for $v=0,1, \ldots, V-1$, and $\widehat{\boldsymbol{Q}}=\left[\begin{array}{llll}\widehat{\boldsymbol{q}}_{0} & \widehat{\boldsymbol{q}}_{1} & \ldots & \widehat{\boldsymbol{q}}_{V-1}\end{array}\right]$.
3) M-ary Baseband Demodulation: Since each sequence $\widehat{\boldsymbol{q}}_{v}$ was originally a pattern of the set $\boldsymbol{P}$, the M-ary baseband demodulator must decide which pattern $\boldsymbol{p}_{h}$ corresponds to each received sequence $\widehat{\boldsymbol{q}}_{v}$. To do so, the correlation between each received sequence $\widehat{\boldsymbol{q}}_{v}$ and each pattern $\boldsymbol{p}_{h}$ is obtained [18] as

$$
\Re_{v, h}=\sum_{c=0}^{31}\left(2 \cdot \widehat{q}_{v, c}-1\right) \cdot\left(2 \cdot p_{h, c}-1\right) .
$$

The pattern $\boldsymbol{p}_{h}$ resulting in the highest correlation with the received sequence $\widehat{\boldsymbol{q}}_{v}$, i.e., $\Re_{v, h}$ fulfilling

$$
\Re_{v, h}>\Re_{v, h^{\prime}} \forall h^{\prime} \neq h,
$$

is considered the correct one and the output $\widehat{\alpha}_{a}$ of the M-ary baseband demodulator for the sequence $\widehat{\boldsymbol{q}}_{v}$ is the RS-symbol associated to the pattern $\boldsymbol{p}_{h}$, i.e., $\widehat{\alpha}_{a}=h$.

When information is transmitted in a double-pulse, two consecutive sequences $\widehat{\boldsymbol{q}}_{v}$ and $\widehat{\boldsymbol{q}}_{v+1}$ are supposed to carry the same pattern. Thus, a pattern $\boldsymbol{p}_{h}$ should fulfil (2) for both received sequences $\widehat{\boldsymbol{q}}_{v}$ and $\widehat{\boldsymbol{q}}_{v+1}$. However, the sequences $\widehat{\boldsymbol{q}}_{v}$ and $\widehat{\boldsymbol{q}}_{v+1}$ may have been differently affected by the channel and their decided patterns may differ. Let us assume that $\boldsymbol{p}_{h}$ fulfils (2) for $\widehat{\boldsymbol{q}}_{v}$ with a correlation value $\Re_{v, h}$. Likewise, $\boldsymbol{p}_{h^{\prime}}$ fulfils (2) for $\widehat{\boldsymbol{q}}_{v+1}$ with a correlation value $\Re_{v+1, h^{\prime}}$. When the JTIDS-type receiver detects that both sequences $\widehat{\boldsymbol{q}}_{v}$ and $\widehat{\boldsymbol{q}}_{v+1}$ come from a double-pulse (by checking the interleaving pattern $\gamma$ ), it outputs a RS-symbol $\widehat{\alpha}_{a}$ given by

$$
\widehat{\alpha}_{a}= \begin{cases}h, & \text { if } h=h^{\prime} \text { or } \Re_{v, h} \geq \Re_{v+1, h^{\prime}} ; \\ h^{\prime}, & \text { if } \Re_{v, h}<\Re_{v+1, h^{\prime}} .\end{cases}
$$

Our way of processing the double-pulses differs from what has been implemented in [18], where both pulses are unconditionally combined during the MSK demodulation. Assuming that the purpose of transmitting the same information in different frequencies is to reduce the probability of both pulses to be affected by the same interference, it seems unlikely that JTIDS receivers employ an unconditional combination of repeated pulses, since then an interfered pulse could spoil its unaffected pair because of the combination. We consider that our implementation should be closer to the mechanism implemented in real JTIDS receivers given that it exploits the frequency diversity gained by using double-pulses whilst not adding any additional block or complex processing algorithm. Note that a more complex processing algorithm is feasible but it should lead to a more robust JTIDS receiver, so the resulting impact of LDACS on JTIDS would be lower than what we obtain in our analysis.

4) Demultiplexing: The output $\widehat{\alpha}$ of the M-ary baseband demodulator block can be decomposed as $\widehat{\boldsymbol{\alpha}}=\left[\widehat{\boldsymbol{\alpha}}^{\mathrm{s}} \widehat{\boldsymbol{\alpha}}^{\mathrm{t}} \widehat{\boldsymbol{\alpha}}^{\mathrm{m}}\right]$. Since perfect synchronization is assumed, $\widehat{\boldsymbol{\alpha}}^{\mathrm{s}}$ and $\widehat{\boldsymbol{\alpha}}^{\mathrm{t}}$ are discarded by the JTIDS-type receiver. The interleaved data and header RS-symbols $\widehat{\boldsymbol{\alpha}}^{\mathrm{m}}$ are forwarded to the de-interleaver.

5) De-interleaving: Using the interleaving pattern $\gamma, \widehat{\boldsymbol{\alpha}}^{\mathrm{m}}$ is de-interleaved. The encoded data $\widehat{\boldsymbol{B}}^{\mathrm{e}}$ and header $\widehat{\boldsymbol{b}}^{\mathrm{h}, \mathrm{e}}$ are forwarded to their respective RS decoders. 
6) RS decoding: The header $\widehat{\boldsymbol{b}}^{\mathrm{h}}$ is obtained by decoding the received $16 \mathrm{RS}$-symbols $\widehat{\boldsymbol{b}}^{\mathrm{h}, \mathrm{e}}$. The $L$ words of data $\widehat{\boldsymbol{B}}$ are obtained by separately decoding each encoded word $\widehat{\boldsymbol{b}}_{l}^{\mathrm{d}, \mathrm{e}}$.

The bit error rate (BER) is used in Section V as a figure of merit to evaluate the impact of LDACS upon JTIDS. The BER of the header is obtained by comparing the received header $\widehat{b}^{\mathrm{h}}$ with the original header $\boldsymbol{b}^{\mathrm{h}}$. Likewise, the BER of the data is obtained by comparing the received data $\widehat{B}$ with the original data $\boldsymbol{B}$. In both cases, the BER can be separately obtained as

$$
\mathrm{BER}=\frac{\text { number of incorrect bits }}{\text { total number of bits }} .
$$

\section{INTERFERENCE SCENARIO}

The simulated interference scenario is shown in Fig. 3. The JTIDS-type transmitter, hereinafter referred to as $\mathrm{JU}_{\mathrm{Tx}}$, transmits a baseband signal $s$ during one time slot. The JTIDS-type receiver, hereinafter referred to as $\mathrm{JU}_{\mathrm{Rx}}$, receives a baseband signal $r$ composed of the desired signal $s$, a noise component $\boldsymbol{n}=\left(n_{0}, n_{1}, \ldots, n_{K-1}\right)^{\mathrm{T}}$, and LDACS interference $\boldsymbol{i}=\left(i_{0}, i_{1}, \ldots, i_{K-1}\right)^{\mathrm{T}}$, such that

$$
r=s+n+i
$$

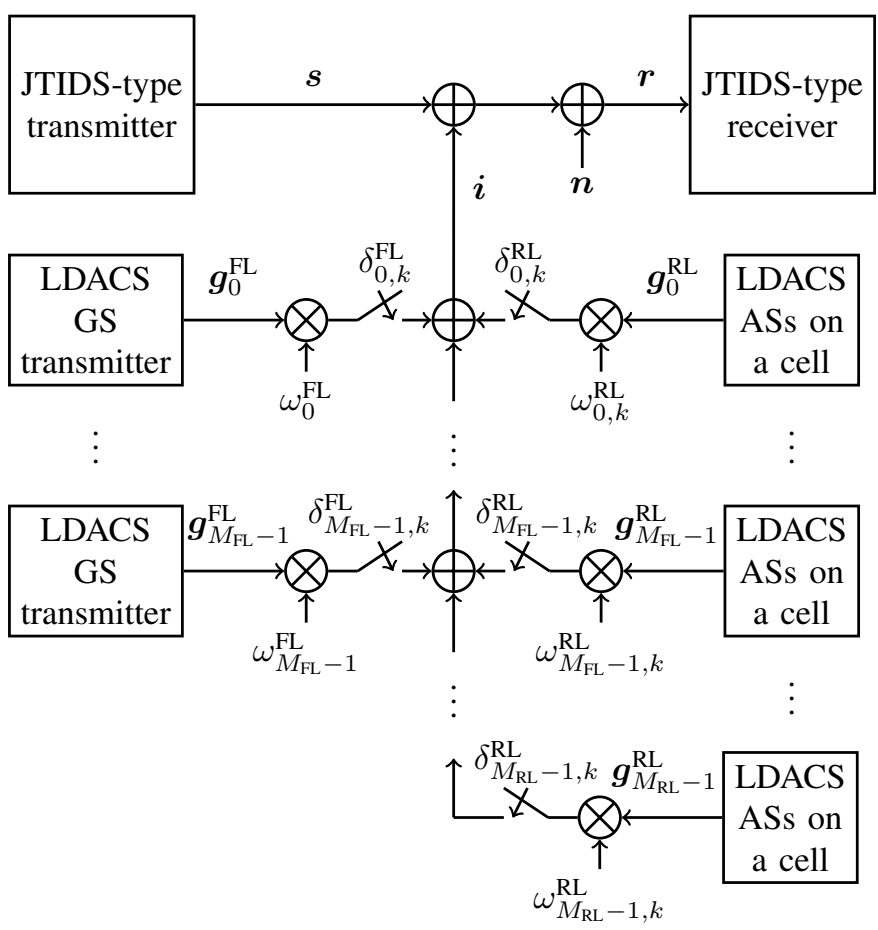

Fig. 3. Interference scenario.

The LDACS interference $i$ is the composed interference originated from radio visible GSs transmitting in the FL and radio visible ASs transmitting in the RL. Let us denote $M_{\mathrm{FL}}$ as the number of GSs with radio visibility to the $\mathrm{JU}_{\mathrm{Rx}}$ and $M_{\mathrm{RL}}$ as the number of LDACS cells where radio visible ASs are operating. The $m_{\mathrm{FL}}-$ th GS generates an LDACS FL baseband signal $\boldsymbol{g}_{m_{\mathrm{FL}}}^{\mathrm{FL}}=$ $\left(g_{m_{\mathrm{FL}}, 0}^{\mathrm{FL}}, g_{m_{\mathrm{FL}}, 1}^{\mathrm{FL}}, \ldots, g_{m_{\mathrm{FL}}, K-1}^{\mathrm{FL}}\right)^{\mathrm{T}}$, for $m_{\mathrm{FL}}=0,1, \ldots, M_{\mathrm{FL}}-1$. Likewise, $\boldsymbol{g}_{m_{\mathrm{RL}}}^{\mathrm{RL}}=\left(g_{m_{\mathrm{RL}}, 0}^{\mathrm{RL}}, g_{m_{\mathrm{RL}}, 1}^{\mathrm{RL}}, \ldots, g_{m_{\mathrm{RL}}, K-1}^{\mathrm{RL}}\right)^{\mathrm{T}}$ contains the tiles transmitted by the ASs operating in the $m_{\mathrm{RL}}$-th LDACS cell, for $m_{\mathrm{RL}}=0,1, \ldots, M_{\mathrm{RL}}-1$. Thus, $\boldsymbol{i}$ represents the joint LDACS interference received by the $\mathrm{JU}_{\mathrm{Rx}}$ and can be obtained as

$i_{k}=\sum_{m_{\mathrm{FL}}=0}^{M_{\mathrm{FL}}-1} \delta_{m_{\mathrm{FL}}, k}^{\mathrm{FL}} \cdot \omega_{m_{\mathrm{FL}}}^{\mathrm{FL}} \cdot g_{m_{\mathrm{FL}}, k}^{\mathrm{FL}}+\sum_{m_{\mathrm{RL}}=0}^{M_{\mathrm{RL}}-1} \delta_{m_{\mathrm{RL}}, k}^{\mathrm{RL}} \cdot \omega_{m_{\mathrm{RL}}, k}^{\mathrm{RL}} \cdot g_{m_{\mathrm{RL}}, k}^{\mathrm{RL}}$,

where $\omega_{m_{\mathrm{FL}}}^{\mathrm{FL}}$ and $\omega_{m_{\mathrm{RL}}}^{\mathrm{RL}}$ model the amplitude ratio between the LDACS signals $\boldsymbol{g}_{m_{\mathrm{FL}}}^{\mathrm{FL}}$ and $\boldsymbol{g}_{m_{\mathrm{RL}}}^{\mathrm{RL}}$, respectively, and the JTIDS signal $s$. Note that $\boldsymbol{\omega}_{m_{\mathrm{RL}}}^{\mathrm{RL}}$ is a vector because the tiles contained in $\boldsymbol{g}_{m_{\mathrm{RL}}}^{\mathrm{RL}}$ are transmitted by different ASs at different distances to the $\mathrm{JU}_{\mathrm{Rx}}$.

Moreover, given that the $\mathrm{JU}_{\mathrm{Rx}}$ changes dynamically its receive frequency following the frequency hopping pattern $\rho$, and frequencies outside its receive bandwidth are filtered out, the signals $\boldsymbol{g}_{m_{\mathrm{FL}}}^{\mathrm{FL}}$ and $\boldsymbol{g}_{m_{\mathrm{RL}}}^{\mathrm{RL}}$ will only affect $s$ when LDACS and JTIDS transmissions coincide in frequency. This frequency selectivity of the $\mathrm{JU}_{\mathrm{Rx}}$ for the signals $\boldsymbol{g}_{m_{\mathrm{FL}}}^{\mathrm{FL}}$ and $\boldsymbol{g}_{m_{\mathrm{RL}}}^{\mathrm{RL}}$ is modelled with the boolean variables $\delta_{m_{\mathrm{FL}}}^{\mathrm{FL}}=\left(\delta_{m_{\mathrm{FL}}, 0}^{\mathrm{FL}}, \delta_{m_{\mathrm{FL}}, 1}^{\mathrm{FL}}, \ldots, \delta_{m_{\mathrm{FL}}, K-1}^{\mathrm{FL}}\right)^{\mathrm{T}}$ and $\delta_{m_{\mathrm{RL}}}^{\mathrm{RL}}=$ $\left(\delta_{m_{\mathrm{RL}}, 0}^{\mathrm{RL}}, \delta_{m_{\mathrm{RL}}, 1}^{\mathrm{RL}}, \ldots, \delta_{m_{\mathrm{RL}}, K-1}^{\mathrm{RL}}\right)^{\mathrm{T}}$, respectively.

In order to estimate $M_{\mathrm{FL}}, M_{\mathrm{RL}}, \omega_{m_{\mathrm{FL}}}^{\mathrm{FL}}$, and $\omega_{m_{\mathrm{RL}}}^{\mathrm{RL}}$, the geographical distribution of the LDACS cells and of the elements of the Link 16 network must be known. Furthermore, the FL and RL frequencies assigned to each LDACS cell are required to estimate $\boldsymbol{\delta}_{m_{\mathrm{FL}}}^{\mathrm{FL}}$ and $\boldsymbol{\delta}_{m_{\mathrm{RL}}}^{\mathrm{RL}}$. However, neither an official cell planning for LDACS has been conducted yet, nor the typical geographical distribution of a Link 16 network has been disclosed. In our analysis, we define a general interference scenario and simulate it for different configurations.

\section{A. Assumed LDACS Deployment}

We approximate the future LDACS deployment with a macrocellular deployment commonly used in mobile communications, where the area of interest is covered by uniformly distributed hexagonal cells and each cell is controlled by a GS [24].

Generically, we consider in our analysis a pseudo-infinite set of cells, each one with a radius $R_{\mathrm{L}}$, covering a pseudoinfinite plane and centred at $\boldsymbol{O}=\left[\begin{array}{llll}\boldsymbol{o}_{0} & \boldsymbol{o}_{1} & \ldots & \boldsymbol{o}_{\infty}\end{array}\right]$. The GS controlling the $j$-th cell is located at the center of the cell, i.e., at $\boldsymbol{o}_{j}=\left(x_{j}, y_{j}, z_{j}\right)^{\mathrm{T}}$, for $j=0,1, \ldots, \infty$. We assume that all GSs have a constant altitude of 15 meters, i.e., $z_{j}=h_{\mathrm{GS}}=15 \mathrm{~m} \forall j$. The ASs registered in the $j^{\text {th }}$ cell are distributed randomly and uniformly around $\boldsymbol{o}_{j}$ at a constant altitude $h_{\mathrm{AS}}=10 \mathrm{~km}$ and at a distance lower than $R_{\mathrm{L}}$ to $\boldsymbol{o}_{j}$. The frequencies assigned to the $j$-th cell are $e_{j}^{\mathrm{FL}}$ for the FL and $e_{j}^{\mathrm{RL}}$ for the RL. These frequencies can be shared with other cells as described afterwards. An exemplary realization of the considered LDACS deployment is illustrated in Fig. 4, where only a part of the pseudo-infinite set $\boldsymbol{O}$ of cells is shown. The separation between adjacent GSs is $d^{\mathrm{g}}$, which is related to the cell radius as $d^{\mathrm{g}}=R_{\mathrm{L}} \cdot \sqrt{3}$ [24]. The cell radius, given by the distance between the centre and the corner of the hexagonal cell, determines the number of GSs needed to cover a certain 
area. LDACS specification [7] considers three different enroute cell radii, $R_{\mathrm{L}} \in\{60,120,200\} \mathrm{nmi}$, which are expected to be covered by the GSs by using an equivalent isotropic radiated power (EIRP), measured in the signal bandwidth, of $E I R P_{\mathrm{GS}} \in\{42,47,52\} \mathrm{dBm}$, respectively. A constant EIRP $E I R P_{\mathrm{AS}}=38.5 \mathrm{dBm}$ is considered for all ASs [7].

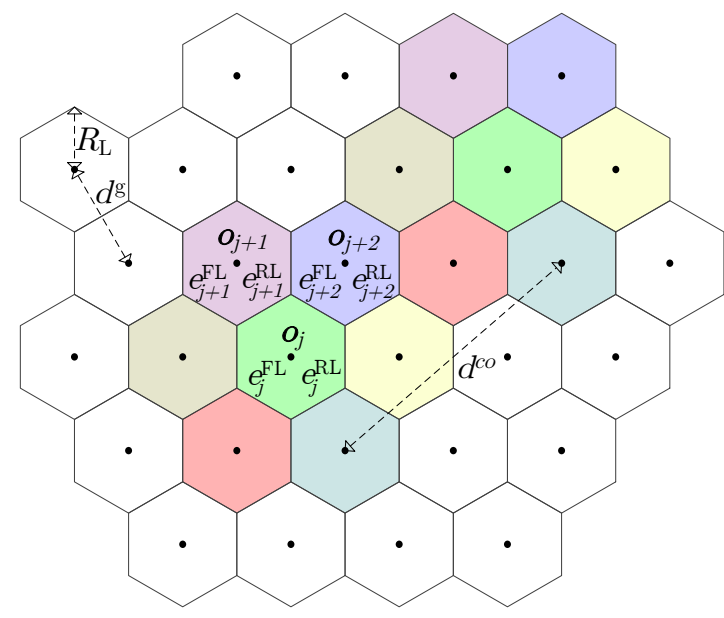

Fig. 4. Exemplary realization of the considered LDACS deployment when $N_{\mathrm{f}}=7$ LDACS FL/RL frequency channels are considered.

Let us assume that LDACS operates using $N_{\mathrm{f}}$ frequencies $\boldsymbol{f}^{\mathrm{FL}}=\left(f_{0}^{\mathrm{FL}}, f_{1}^{\mathrm{FL}}, \ldots, f_{N_{\mathrm{f}}-1}^{\mathrm{FL}}\right)^{\mathrm{T}}$ for the $\mathrm{FL}$ and $N_{\mathrm{f}}$ frequencies $f^{\mathrm{RL}}=\left(f_{0}^{\mathrm{RL}}, f_{1}^{\mathrm{RL}}, \ldots, f_{N_{\mathrm{f}}-1}^{\mathrm{RL}}\right)^{\mathrm{T}}$ for the RL. The frequencies $e_{j}^{\mathrm{FL}}$ and $e_{j}^{\mathrm{RL}}$ are assigned to the $j^{\text {th }}$ cell from the sets of available frequencies $f^{\mathrm{FL}}$ and $f^{\mathrm{RL}}$, i.e., $e_{j}^{\mathrm{FL}} \in f^{\mathrm{FL}}$ and $e_{j}^{\mathrm{RL}} \in \boldsymbol{f}^{\mathrm{RL}}$. Likewise, a pair of FL and RL frequencies must be assigned to each cell by following a careful frequency planing where many different factors must be taken into account, such as the distance between cells using the same frequency or the interference with other systems. In our analysis, following the typical frequency planning used in macrocellular deployments [24], we consider a simplified LDACS frequency planning where clusters of $N_{\mathrm{f}}$ neighbouring cells are formed. The $N_{\mathrm{f}}$ frequencies $f^{\mathrm{FL}}$ and the $N_{\mathrm{f}}$ frequencies $f^{\mathrm{RL}}$ are distributed among the $N_{\mathrm{f}}$ cells forming each cluster, following a common frequency distribution pattern to guarantee that the minimum distance between GSs using the same frequency is $d^{\text {co }}=$ $R_{\mathrm{L}} \cdot \sqrt{3 \cdot N_{\mathrm{f}}}$ [24]. An example of such a repetitive frequency distribution pattern is shown in Fig. 4 for the case $N_{\mathrm{f}}=7$, where each color represents a different FL/RL frequency channel assigned to a cell.

The number of frequency channels that will be available for the operation of LDACS has not yet been determined. Nevertheless, according to the results presented in [25] and [26] regarding the feasibility of LDACS cell planning in Europe, up to 12 frequency channels might be enough for the LDACS FL to support air traffic growth in Europe. Therefore, $N_{\mathrm{f}}=12$ is expected to be a reasonable assumption. In order to keep our analysis as general as possible, we also analyse the effect of decreasing and increasing $N_{\mathrm{f}}$ around its expected value. Hence, we consider $N_{\mathrm{f}} \in\{7,12,16\}$.

LDACS will operate within the frequency bands 1110-1156 $\mathrm{MHz}$ for the FL and 964-1010 MHz for the RL [20]. Since no
DME stations operate in the lowest part of the RL frequency band [20], it is expected that most LDACS cells will use the lowest RL frequency channels. In addition, the Resolution 417 of the WRC-2015 [27] establishes a power limitation for LDACS stations that forces them to operate in the lowest part of the FL frequency band, i.e., practically below 1127 $\mathrm{MHz}$ for the considered $E I R P_{\mathrm{GS}}$ or at higher frequencies if the EIRP is drastically reduced. Therefore, we assume for our analysis $f^{\mathrm{FL}}=\left(1110.5,1111.5, \ldots, 1110.5+N_{\mathrm{f}}-1\right)$ and $f^{\mathrm{RL}}=\left(964.5,965.5, \ldots, 964.5+N_{\mathrm{f}}-1\right)$. Note that, even though an LDACS channel grid of $0.5 \mathrm{MHz}$ could be used [20], we assume a channel grid of $1 \mathrm{MHz}$ to cover a wider frequency band and so to affect more JTIDS channels. As all JTIDS channels are used with the same probability, affecting more JTIDS channels should lead to a higher impact of LDACS on JTIDS.

The GS controlling each cell assigns dynamically and under demand the available tiles to the ASs. Therefore, the LDACS RL duty cycle $D$ will change from multi-frame to multi-frame and from cell to cell. It will depend, among other factors, on the traffic density within the cell, the cell radius, and the needs of the ASs. A worst-case RL duty cycle $D_{\mathrm{wc}}=1$, i.e., all tiles are used, will only happen in reality in very congested cells and/or during very short periods of time. Therefore, it is not realistic to use the worst-case RL duty cycle for all cells of $\boldsymbol{O}$. In order to find a realistic duty cycle for our analysis, we simulate the "En-route medium", "En-route large", and "Enroute super large" air traffic volumes defined in [28] with the ATS+AOC data traffic profile defined in the COCRv2 report [29]. Simulations are repeated for different cell sizes and the RL duty cycle is measured for each multi-frame. The resulting cumulative distribution function of the RL duty cycle is shown in Fig. 5. One can see that the $90 \%$ percentile of the measured RL duty cycle is $0.45,0.48$, and 0.51 , for a cell radius of 60 $\mathrm{nmi}, 120 \mathrm{nmi}$, and $200 \mathrm{nmi}$, respectively. As a conservative assumption for a higher impact of LDACS on JTIDS, we take $D=0.5$ for all cells of $\boldsymbol{O}$ and for the entire simulation time. In addition, we assume that each assigned full-tile is transmitted by a different AS.

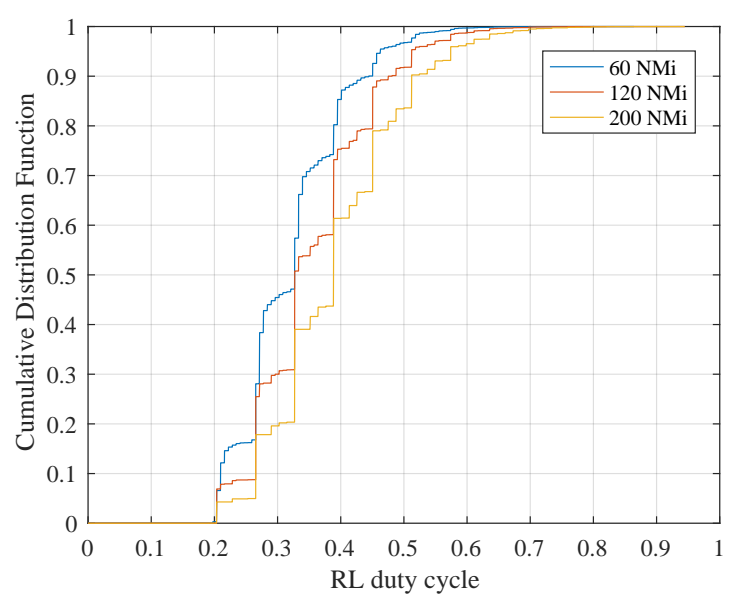

Fig. 5. Cumulative distribution function of the LDACS RL duty cycle for different LDACS cell radii. 


\section{B. Assumed Link 16 Network}

We consider that a Link 16 network has been established in an area where LDACS is deployed as described in Section IV-A. In our analysis, we consider that the Link 16 network may be composed of several Link 16 subnetworks, but we assume that their frequency hopping patterns effectively avoid interference between them.

Given that each frequency hopping pattern must use all the JTIDS frequencies with the same probability, the impact of LDACS on every Link 16 subnetwork is expected to be, on average, the same. Consequently, in our analysis, we only consider the impact of LDACS on one Link 16 subnetwork, which can be considered representative of the impact of LDACS on the entire Link 16 network. Thus, we consider a Link 16 subnetwork composed of a transmitter $\mathrm{JU}_{\mathrm{Tx}}$ and a receiver $\mathrm{JU}_{\mathrm{Rx}}$. The $\mathrm{JU}_{\mathrm{Tx}}$ transmits in all available time slots and the $\mathrm{JU}_{\mathrm{Rx}}$ receives and processes all transmissions. Note that, given that a dedicated access to the time slots is assumed, this scenario is equivalent to the scenario where different transmitters use all available time slots and the power of the signal received by every receiver is always the same.

The JTIDS signal $s$ is received by the $\mathrm{JU}_{\mathrm{Rx}}$ with an average power $P^{\mathrm{J}}$ and affected by a zero-mean additive white gaussian noise (AWGN) component $\boldsymbol{n}$ with a variance $\sigma_{n}^{2}=\frac{N_{0}}{2}$, such that $n_{k} \in \mathcal{N}\left(0, \sigma_{n}^{2}\right)$. The noise power $N$ can be obtained as

$$
N[\mathrm{~dB}]=10 \cdot \log _{10}\left(k_{B} \cdot T\right)+10 \cdot \log _{10}\left(B W_{\mathrm{Rx}}\right)+F,
$$

where $k_{B}$ is the Boltzmann constant, $T$ the receiver temperature, $B W_{\mathrm{Rx}}$ the receiver bandwidth, and $F$ the receiver noise figure. We assume $T=290 \mathrm{~K}$ and $F=10 \mathrm{~dB}$, which are typical values for L-band airborne receivers. The value of $B W_{\mathrm{Rx}}$ is discussed in Section IV-C2.

For our analysis, we consider different values of the signalto-noise ratio (SNR), which can be obtained as

$$
S N R[\mathrm{~dB}]=P^{\mathrm{J}}-N \text {. }
$$

Different SNR values are simulated by generating a noise component $\boldsymbol{n}$ with an average power $S N R$ dB below the average power of $s$.

In a real scenario, the interference conditions between LDACS and JTIDS will vary continuously depending on the locations of the LDACS platforms, the $\mathrm{JU}_{\mathrm{Tx}}$, and the $\mathrm{JU}_{\mathrm{Rx}}$. For our analysis, we consider different locations and altitudes of the $\mathrm{JU}_{\mathrm{Rx}}$ relative to the locations of the GSs. For each location and altitude, a different impact of LDACS on JTIDS is obtained. We expect that, in a real scenario, the average impact will vary within the range of impacts obtained in our analysis for the different locations and altitudes.

We consider that the $\mathrm{JU}_{\mathrm{Rx}}$ is located at $\left(x^{\mathrm{Rx}}, y^{\mathrm{Rx}}, z^{\mathrm{Rx}}\right)$ and flying over the pseudo-infinite plane where the LDACS cells $\boldsymbol{O}$ are located. Three different altitudes of the $\mathrm{JU}_{\mathrm{Rx}}$ are considered in the simulations: $z^{\mathrm{Rx}}=h_{\mathrm{Rx}} \in\{3.048,10,18.288\} \mathrm{km}$. In addition, three different locations relative to the GSs are considered:

- Location A: The $\mathrm{JU}_{\mathrm{Rx}}$ is flying over the GS located at $\boldsymbol{o}_{j}$, as given by

$$
\left.\left(x^{\mathrm{Rx}}, y^{\mathrm{Rx}}, z^{\mathrm{Rx}}\right)\right|_{A}=\left(x_{j}, y_{j}, h_{\mathrm{Rx}}\right) .
$$

- Location B: The $\mathrm{JU}_{\mathrm{Rx}}$ is flying over the border between three adjacent cells located at $\boldsymbol{o}_{j}, \boldsymbol{o}_{j+1}$ and $\boldsymbol{o}_{j+2}$, such that

$$
\begin{gathered}
\left.\left(x^{\mathrm{Rx}}, y^{\mathrm{Rx}}, z^{\mathrm{Rx}}\right)\right|_{B}= \\
\left(\frac{x_{j}+x_{j+1}+x_{j+2}}{3}, \frac{y_{j}+y_{j+1}+y_{j+2}}{3}, h_{\mathrm{Rx}}\right) .
\end{gathered}
$$

- Location C: The $\mathrm{JU}_{\mathrm{Rx}}$ is flying over the border between two adjacent cells located at $\boldsymbol{o}_{j}$ and $\boldsymbol{o}_{j+1}$, on the closest point between both GSs, as given by

$$
\left.\left(x^{\mathrm{Rx}}, y^{\mathrm{Rx}}, z^{\mathrm{Rx}}\right)\right|_{C}=\left(\frac{x_{j}+x_{j+1}}{2}, \frac{y_{j}+y_{j+1}}{2}, h_{\mathrm{Rx}}\right) .
$$

\section{Interference Conditions}

1) Radio Visibility: Only transmissions from radio visible LDACS stations are considered. For radio line-of-sight (RLOS) communications and only taking into account the earth curvature, it has been shown that the distance between a station located at an altitude $z^{\mathrm{Tx}}$ and an aircraft flying at an altitude $h_{\mathrm{Rx}}$ must be lower than [30]

$$
R_{\operatorname{los}}[k m]=130.4\left(\sqrt{z^{\mathrm{Tx}}[k m]}+\sqrt{h_{\mathrm{Rx}}[k m]}\right),
$$

when considering the earth radius to be $R_{\mathrm{e}}=6378.137 \mathrm{~km}$ and scaling it by a factor of $\frac{4}{3}$ to account for the refraction in the atmosphere.

Therefore, the $\mathrm{JU}_{\mathrm{Rx}}$ receives the transmissions from an LDACS station located at $\left(x^{\mathrm{Tx}}, y^{\mathrm{Tx}}, z^{\mathrm{Tx}}\right)$ only if

$$
d \leq R_{\mathrm{los}}
$$

is fulfilled, where $d$ is the distance between both platforms and can be obtained as

$$
d=\sqrt{\left(x^{\mathrm{Rx}}-x^{\mathrm{Tx}}\right)^{2}+\left(y^{\mathrm{Rx}}-y^{\mathrm{Tx}}\right)^{2}+\left(h_{\mathrm{Rx}}-z^{\mathrm{Tx}}\right)^{2}} .
$$

Note that $R_{\text {los }}$ is greater for ASs, where $z^{\mathrm{Tx}}=h_{\mathrm{AS}}=10 \mathrm{~km}$, than for GSs, where $z^{\mathrm{Tx}}=h_{\mathrm{GS}}=15 \mathrm{~m}$. Consequently, some radio visible ASs will be registered to cells whose GSs are beyond RLOS. Specifically, radio visible ASs can be registered to any cell controlled by a GS fulfilling

$$
d \leq R_{\mathrm{los}}^{\mathrm{as}}+R_{\mathrm{L}},
$$

where $R_{\text {los }}^{\mathrm{as}}$ is obtained using (8) for $z^{\mathrm{Tx}}=h_{\mathrm{AS}}=10 \mathrm{~km}$. Thus, out of the original set of cells $\boldsymbol{O}$, the $\mathrm{JU}_{\mathrm{Rx}}$ receives interference from $M_{\mathrm{FL}}$ radio visible GSs, i.e., GSs fulfilling (9), and from the radio visible ASs, i.e., ASs fulfilling (9), registered to the $M_{\mathrm{RL}}$ cells fulfilling (11).

Generally, let us say that the $k$-th sample of the $m$-th LDACS signal, i.e., $g_{m, k}$, received by the $\mathrm{JU}_{\mathrm{Rx}}$ is transmitted by a radio visible LDACS station separated a distance $d_{m, k}$ and using a frequency $e_{m}$. The power of the received signal can be estimated as

$$
\begin{aligned}
P_{m, k}^{\mathrm{L}}[\mathrm{dB}]= & E I R P[\mathrm{dBm}]-30 \mathrm{~dB}+G_{\mathrm{Rx}}[\mathrm{dBi}]-32.44 \mathrm{~dB} \\
& -20 \log _{10}\left(d_{m, k}[\mathrm{~km}]\right)-20 \log _{10}\left(e_{m}[\mathrm{MHz}]\right),
\end{aligned}
$$

where free-space path losses are considered and EIRP is $E I R P_{\mathrm{GS}}$ for a GS and $E I R P_{\mathrm{AS}}$ for an AS. $G_{R x}$ represents 
the joint effect of the $\mathrm{JU}_{\mathrm{Rx}}$ antenna gain and cable losses. We assume $G_{R x}=0 \mathrm{dBi}$, which is a conservative assumption for a higher impact of LDACS on JTIDS given that the cable losses are usually higher than the antenna gain in airborne platforms, e.g., as assumed for LDACS airborne receivers in [7]. Note that, in addition, we assume the direction of maximum antenna gain for all LDACS stations, which also represents a worstcase assumption for a stronger interference of LDACS on JTIDS.

2) Frequency Selectivity: No information has been found regarding the frequency selectivity of a JTIDS receiver. In our analysis, we assume an ideal receive filter with a passband bandwidth $B W_{\mathrm{Rx}}$. Thus, when the $\mathrm{JU}_{\mathrm{Rx}}$ tunes into the carrier frequency $\rho_{k}$, only LDACS transmissions at a frequency $e_{m}$ fulfilling

$$
\left|\rho_{k}-e_{m}\right| \leq \frac{B W_{\mathrm{Rx}}}{2}
$$

are processed in the baseband together with the received JTIDS pulse.

Thus, $\delta_{m, k}$ can be obtained as

$$
\delta_{m, k}=\left\{\begin{array}{ll}
1, & \text { if }\left|\rho_{k}-e_{m}\right| \leq \frac{B W_{\mathrm{Rx}}}{2} \\
0, & \text { otherwise }
\end{array} .\right.
$$

Given that adjacent JTIDS carrier frequencies are separated by $3 \mathrm{MHz}$, a $B W_{\mathrm{Rx}}$ of $3 \mathrm{MHz}$ could be considered. However, since a $3 \mathrm{MHz}$ bandwidth filtering seems too demanding for a real implementation, we assume $B W_{\mathrm{Rx}}=4 \mathrm{MHz}$. By considering $B W_{\mathrm{Rx}}=4 \mathrm{MHz}$ and taking into account the offset between the LDACS carrier frequencies $f^{\mathrm{FL}}$ and $f^{\mathrm{RL}}$, and the JTIDS carrier frequencies $\boldsymbol{f}^{\mathrm{J}}$, we are assuming in our analysis that a single JTIDS channel can be interfered by transmissions from up to 4 different LDACS channels at the same time, located at offsets of $\pm 0.5 \mathrm{MHz}$ and $\pm 1.5 \mathrm{MHz}$ to the JTIDS carrier frequency. For example, the JTIDS channel centred at $1116 \mathrm{MHz}$ is affected by transmissions in the LDACS channels centred at $1114.5 \mathrm{MHz}, 1115.5 \mathrm{MHz}, 1116.5 \mathrm{MHz}$ and $1117.5 \mathrm{MHz}$. Moreover, an LDACS channel centred at an offset of $\pm 1.5 \mathrm{MHz}$ to two adjacent JTIDS channels interferes receptions in both JTIDS channels equally. Using the previous example, LDACS transmissions using the LDACS channel centred at $1117.5 \mathrm{MHz}$ interfere pulses received at the JTIDS frequencies $1116 \mathrm{MHz}$ and $1119 \mathrm{MHz}$.

3) Desired-to-undesired Power Ratio: When the $\mathrm{JU}_{\mathrm{Rx}}$ receives a pulse at a frequency $\rho_{k}$ and (13) is fulfilled for an LDACS station transmitting at a frequency $e_{m}$, the desired-toundesired $(\mathrm{D} / \mathrm{U})$ power ratio between the desired JTIDS pulse and the undesired LDACS signal is given by

$$
D / U_{m, k}=P^{\mathrm{J}}-P_{m, k}^{\mathrm{L}}=S N R+N-P_{m, k}^{\mathrm{L}} .
$$

where $N$ and $P_{m, k}^{\mathrm{L}}$ can be obtained using (6) and (12), respectively.

Thus, the amplitude scaling factor $\omega_{m, k}$ can be obtained as

$$
\omega_{m, k}=\sqrt{\frac{\frac{1}{K_{\mathrm{on}}} \sum_{k=0}^{K-1}\left|s_{k}\right|^{2}}{\frac{1}{K} \sum_{k=0}^{K-1}\left|g_{m, k}\right|^{2}} 10^{-\frac{D / U_{m, k}}{10}}},
$$

where $K_{\mathrm{on}}=\frac{6.4 \mu \mathrm{s}}{0.2 \mu s} \mathrm{~V}$ is the number of samples of $s$ where real transmission takes place during a time slot, i.e., the samples corresponding to the active part of the pulses.

Note that, as the FL interference from the $m_{\mathrm{FL}}$-th cell is only transmitted by a GS, with a constant distance $d_{m_{\mathrm{FL}}}$ to the $\mathrm{JU}_{\mathrm{Rx}}$, $D / U_{m_{\mathrm{FL}}, k}=D / U_{m_{\mathrm{FL}}} \forall k$. By contrast, the RL interference coming from the $m_{\mathrm{RL}}$-th cell is composed of multiple fulltiles generated by different ASs at different distances $d_{m_{\mathrm{FL}}, k}$. As we assume that every full-tile is transmitted by a different AS, $D / U_{m_{\mathrm{RL}}, k}$ changes every $\frac{0.72 \mu \mathrm{ms}}{0.2 \mu \mathrm{s}}$ samples.

\section{Simulation of the Interference Scenario}

In our analysis, we simulate the interference scenario for a wide variety of configurations, given by the different values of $R_{\mathrm{L}}, E I R P_{\mathrm{GS}}, N_{\mathrm{f}},\left(x^{\mathrm{Rx}}, y^{\mathrm{Rx}}, h_{\mathrm{Rx}}\right), D$, and $S N R$. For each configuration, the interference scenario is simulated using a series of steps.

1) The implemented JTIDS-type transmitter generates $s$ and $\rho$,

2) the noise component $\boldsymbol{n}$ is generated for the desired $S N R$,

3) the locations $\boldsymbol{O}$ and frequencies $e^{\mathrm{FL}}$ and $e^{\mathrm{RL}}$ of the LDACS cells are computed taking into account $R_{\mathrm{L}}$ and $N_{\mathrm{f}}$,

4) $\delta_{m_{\mathrm{FL}}, k}^{\mathrm{FL}}$ and $\omega_{m_{\mathrm{FL}}}^{\mathrm{FL}}$ are obtained using (14) and (16), respectively, for each one of the $M_{\mathrm{FL}}$ GSs fulfilling (9) for the given $\left(x^{\mathrm{Rx}}, y^{\mathrm{Rx}}, h_{\mathrm{Rx}}\right)$, i.e., for $m_{\mathrm{FL}}=0,1, \ldots, M_{\mathrm{FL}}-$ 1 ,

5) $M_{\mathrm{FL}}$ FL LDACS baseband signals $\boldsymbol{g}_{m_{\mathrm{FL}}}^{\mathrm{FL}}$ are generated,

6) for each one of the $M_{\mathrm{RL}}$ cells fulfilling (11) for the given $\left(x^{\mathrm{Rx}}, y^{\mathrm{Rx}}, h_{\mathrm{Rx}}\right)$, i.e., for $m_{\mathrm{RL}}=0,1, \ldots, M_{\mathrm{RL}}-1$,

a) $\delta_{m_{\mathrm{RL}}, k}^{\mathrm{RL}}$ is obtained using (14),

b) the locations of the ASs are generated randomly and uniformly within the cell limits,

c) the distance from each ASs to the $\mathrm{JU}_{\mathrm{Rx}}$ is computed using (10) and $\omega_{m_{\mathrm{RL}}, k}^{\mathrm{RL}}$ is obtained using (16),

d) the $\omega_{m_{\mathrm{RL}}, k}^{\mathrm{RL}}$ corresponding to any transmission from an AS not fulfilling (9) is set to 0,

7) $M_{\mathrm{RL}}$ RL LDACS baseband signals $\boldsymbol{g}_{m_{\mathrm{RL}}}^{\mathrm{RL}}$ with a duty cycle $D$ are generated,

8) $i$ is obtained using (5),

9) $\boldsymbol{r}$ is obtained using (4),

10) $r$ is processed by the implemented JTIDS-type receiver and the BER is computed.

Note that we simplify our analysis by considering that full-tiles transmitted from different ASs within the same cell are received synchronously by the $\mathrm{JU}_{\mathrm{Rx}}$, i.e., they do not overlap with each other, which is only true if the $\mathrm{JU}_{\mathrm{Rx}}$ is colocated with the GS controlling the cell. However, this simplification reduces significantly the analysis complexity and is not expected to noticeably affect the results. Nevertheless, transmissions from ASs located in different cells are received asynchronously by the $\mathrm{JU}_{\mathrm{Rx}}$.

\section{E. Propagation Channel}

As shown in Fig. 3, we consider in our analysis an ideal channel impulse response, where no multipath components are 
present and, therefore, the received signal is not affected by fading. This decision is based on the following points. Firstly, results might depend strongly on the specifics of the chosen channel model. Therefore, an appropriate channel model would be necessary in order to trustfully cover a wide variety of cases. However, no studies have been found regarding realistic channel models applicable to JTIDS operations. In addition, typical JTIDS operations have not been disclosed and, therefore, a realistic channel model cannot be trustfully extrapolated from the air-air and air-ground channel models currently present in the literature. Moreover, using an idealized channel model as the one shown in Fig. 3 allows us to isolate the effect of the LDACS interference on JTIDS transmissions and, therefore, to conduct a more general analysis. Secondly, the presence of fading would increase the minimum SNR required by JTIDS to maintain a certain BER compared to the case without fading. Considering now the LDACS interference, such increase in the required SNR or, equivalently, in the required JTIDS received signal power, would lead to a higher D/U power ratio (see Eq. (15)). Given that a higher $\mathrm{D} / \mathrm{U}$ power ratio yields a JTIDS transmission less sensitive to LDACS interference, the impact of LDACS on JTIDS would be reduced, compared to the case without fading. In addition, as LDACS signals would also undergo fading, the D/U power ratio would be further increased. Consequently, we do not consider fading in our analysis as a conservative assumption for a higher impact of LDACS on JTIDS.

\section{Simulation Results}

The scenario defined in Section IV has been simulated for different interference configurations as described in Section IV-D. The obtained results are presented in this section. Each configuration has been simulated iteratively until the resulting statistics reached a stable point. In each iteration, the $\mathrm{JU}_{\mathrm{Tx}}$ transmits 1000 messages using 1000 consecutive time slots. A different frequency hopping pattern fulfilling the frequency hopping conditions described in Section III is randomly generated for each iteration.

No official criteria have been found in the literature to assess the degree of degradation of JTIDS in the presence of external interference. In our analysis, we assume that the correct operation of JTIDS depends strongly on the BER of the information bits received by the addressed JTIDS platform. Given that the BER depends on the SNR, we use the relation between the BER and the SNR as a figure of merit to assess the impact of LDACS on JTIDS.

First, we obtain the relation between the SNR and the BER when no LDACS interference affects the JTIDS transmission, i.e., when $i_{k}=0 \forall k$. The obtained relation represents the baseline of the performance of JTIDS. Secondly, the relation between the SNR and the BER is obtained for different configurations of the interference scenario. By comparing the performance of JTIDS obtained with LDACS interference, with the baseline obtained without LDACS interference, the degradation of JTIDS in the presence of LDACS is quantified as the increase in SNR needed to achieve the same BER, which can be seen as the SNR loss experienced by JTIDS because of the presence of LDACS.

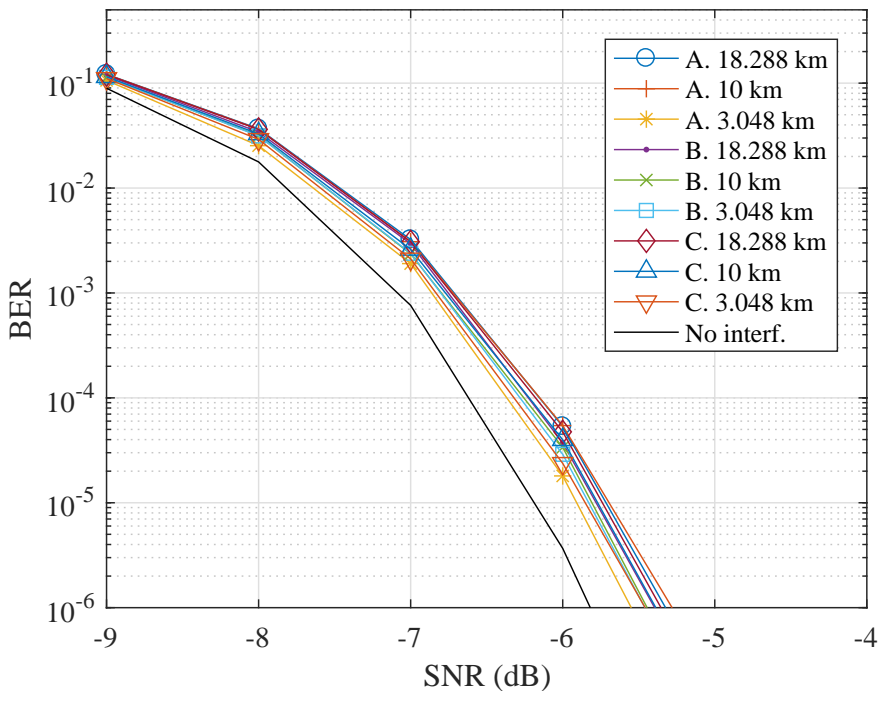

Fig. 6. Bit error rate of the data in STD messages. Without LDACS interference ("No interf." curve) and with LDACS interference (other curves). $N_{\mathrm{f}}=12 . R_{\mathrm{L}}=120 \mathrm{nmi} . E I R P_{\mathrm{GS}}=47 \mathrm{dBm} . D=0.5$.

Simulations are run for the expected LDACS deployment using the medium cell size, i.e., $R_{\mathrm{L}}=120 \mathrm{nmi}$ and $E I R P_{\mathrm{GS}}=47 \mathrm{dBm}$. In addition, the expected number of frequencies $N_{\mathrm{f}}=12$ and a conservative RL duty cycle $D=0.5$ are used. Figure 6 shows the SNR needed to get a certain BER of the bits of the data when STD messages are used. The interference-free performance is shown in the curve labelled as "No interf.". The other 9 curves represent the results obtained for the combination of the considered flying altitudes and locations of the $\mathrm{JU}_{\mathrm{Rx}}$. It can be seen that, when LDACS interference is present, the SNR needed to achieve a certain BER is increased, compared to the case without LDACS interference. In the worst case obtained, i.e., when the $\mathrm{JU}_{\mathrm{Rx}}$ is flying directly over a GS at a high altitude, the SNR needed to maintain $\mathrm{BER}=10^{-6}$ increases from $S N R=-5.82 \mathrm{~dB}$ without LDACS interference, to $S N R=-5.28 \mathrm{~dB}$ with LDACS interference. Therefore, the maximum degradation for this configuration is $\Delta S N R=0.54$ $\mathrm{dB}$. The lowest degradation for this configuration is obtained when the $\mathrm{JU}_{\mathrm{Rx}}$ is flying over a GS at a low altitude $h=3.048$ $\mathrm{km}$. In this case, an increase of $\Delta S N R=0.27 \mathrm{~dB}$ is needed to maintain BER $=10^{-6}$ when LDACS interference is present. Thus, for this configuration of the interference scenario, as the $\mathrm{JU}_{\mathrm{Rx}}$ flies over an area covered by GSs, the degradation of a JTIDS data transmission using STD messages is expected to oscillate within the range $\triangle S N R \in[0.27,0.54] \mathrm{dB}$ for $\mathrm{BER}=10^{-6}$.

Results shown in Fig. 6 are also valid for data transmitted using P2DP messages, given that data are encoded in the same way and transmitted in double-pulses in both message structures. The differences between STD and P2DP messages, as the net data throughput and jitter, do not affect the relation between the BER and the SNR in the defined interference scenario.

Figure 7 shows the results obtained when data are transmitted in P2SP messages. It can be seen that the SNR loss in this 


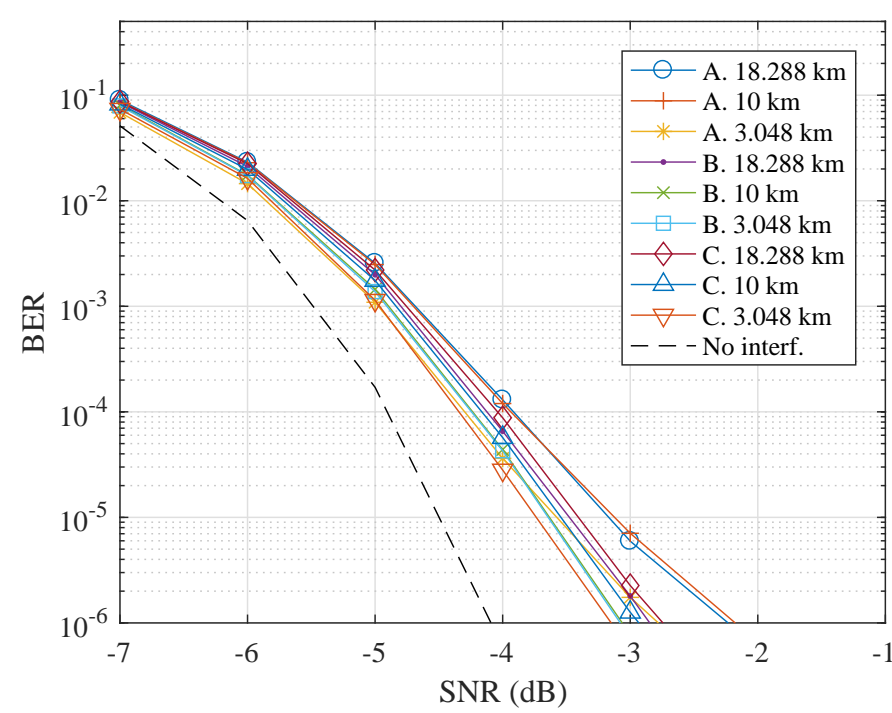

Fig. 7. Bit error rate of the data in P2SP messages. Without LDACS interference ("No interf." curve) and with LDACS interference (other curves). $N_{\mathrm{f}}=12 . R_{\mathrm{L}}=120 \mathrm{nmi}$. $E I R P_{\mathrm{GS}}=47 \mathrm{dBm} . D=0.5$.

case oscillates within the range $\Delta S N R \in[0.92,1.95] \mathrm{dB}$ for $\mathrm{BER}=10^{-6}$ as the $\mathrm{JU}_{\mathrm{Rx}}$ flies over an area where LDACS is deployed. Note that the simulation results shown in Fig. 7 are also valid for data transmitted using P4SP messages.

Figure 8 shows the relation between the BER of the bits of the header and the SNR. With a degradation within the range $\Delta S N R \in[0.33,0.60] \mathrm{dB}$ for $\mathrm{BER}=10^{-6}$, it can be seen that the header is only slightly more affected by LDACS interference than data transmitted in double-pulses. Given that the header is always equally protected, results shown in Fig. 8 do not depend on the message structure used by the transmitter.

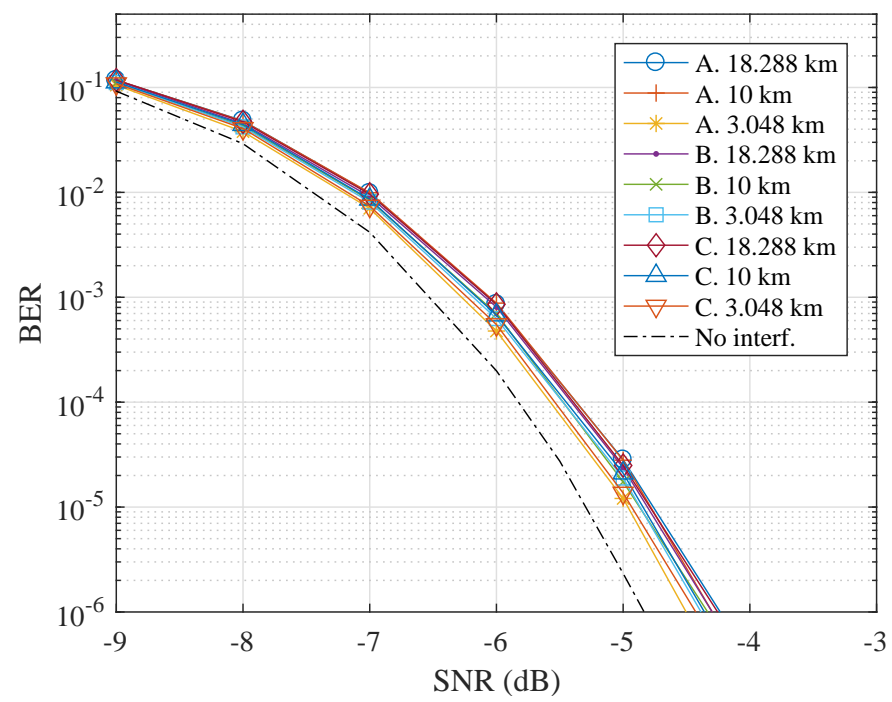

Fig. 8. Bit error rate of the header. Without LDACS interference ("No interf." curve) and with LDACS interference (other curves). $N_{\mathrm{f}}=12 . R_{\mathrm{L}}=120$ nmi. $E I R P_{\mathrm{GS}}=47 \mathrm{dBm} . D=0.5$.

From the obtained results, one can see that JTIDS transmissions in single-pulses are more affected by LDACS interference than transmissions in double-pulses. In any case, however, it can be seen that the degradation depends on the position of the $\mathrm{JU}_{\mathrm{Rx}}$, being minimum for low flying altitudes and maximum when the $\mathrm{JU}_{\mathrm{Rx}}$ flies directly over a GS at a high altitude. This variation is specially noticeable for singlepulse transmissions, where the SNR loss experienced by the $\mathrm{JU}_{\mathrm{Rx}}$ when it flies directly over a GS at a high altitude is, at least, $0.5 \mathrm{~dB}$ higher than in any other case. However, it must be taken into account that, in a real LDACS deployment, a GS antenna will provide its maximum gain at low elevation angles, in order to be able to serve ASs at the border of the cell. Consequently, the LDACS signal power that the $\mathrm{JU}_{\mathrm{Rx}}$ will receive in reality when flying directly over a GS, i.e., at high elevation angles, will be significantly lower than the value assumed here. Therefore, the maximum SNR loss obtained in our analysis is expected to be lower in reality and closer to the SNR loss obtained for the other considered locations.

It is to be noted that the SNR required to achieve BER = $10^{-6}$ for both the header and the data transmitted in doublepulses is, even under LDACS interference, lower than the SNR required to achieve $\mathrm{BER}=10^{-6}$ for data transmitted in singlepulses without LDACS interference.

As transmissions in double-pulses are not strongly affected by LDACS interference, the obtained results strengthen the assumption made in our analysis of all JTIDS platforms remaining synchronized despite LDACS interference, given that synchronization and time refinement information are always transmitted using double-pulses.

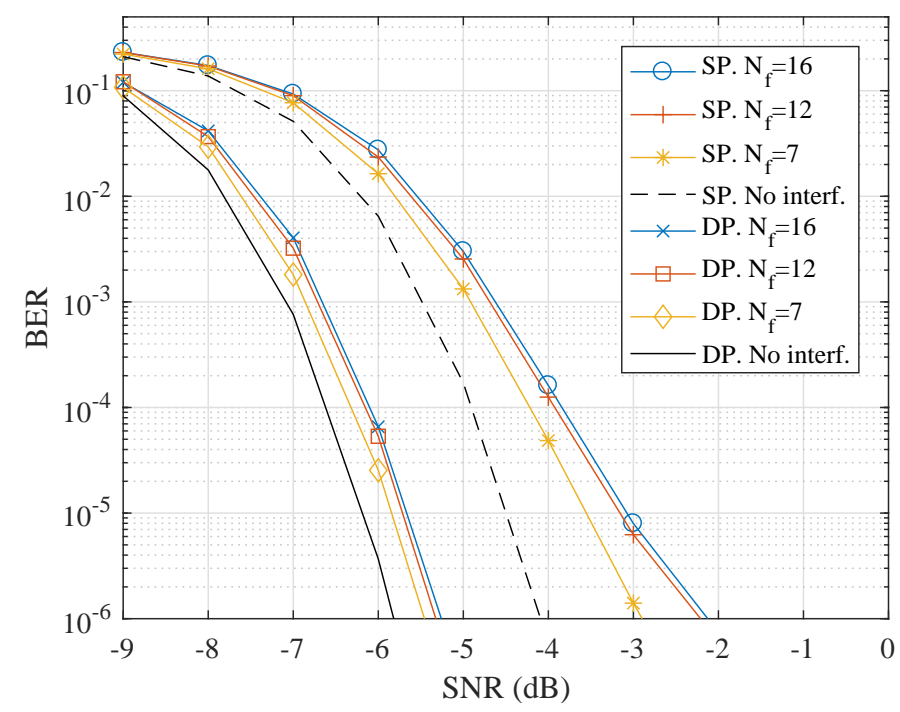

Fig. 9. Bit error rate of the data in P2SP (labelled as "SP") and STD messages (labelled as "DP") for a different number of frequency channels $N_{\mathrm{f}}$. Without LDACS interference ("No interf." curves) and with LDACS interference (other curves). $R_{\mathrm{L}}=120 \mathrm{nmi}$. $E I R P_{\mathrm{GS}}=47 \mathrm{dBm} . D=0.5$.

In order to evaluate how a variation in the number of LDACS frequency channels around its expected value might affect the impact of LDACS on JTIDS, simulations are repeated for $N_{\mathrm{f}}=7$ and $N_{\mathrm{f}}=16$. For simplicity, we simulate the transmission of data in STD and P2SP message structures when the $\mathrm{JU}_{\mathrm{Rx}}$ is flying at an altitude of $18.288 \mathrm{~km}$ directly over a GS. Simulation results are shown jointly in Fig. 9. Compared to the results obtained for the expected number of frequency channels $N_{\mathrm{f}}=12$, one can see that adding 4 frequency channels affects marginally data transmissions in 
either double-pulses or single-pulses, with an increase in the maximum SNR loss of $0.07 \mathrm{~dB}$ and $0.09 \mathrm{~dB}$, respectively. By contrast, even though removing 5 frequency channels reduces by only $0.13 \mathrm{~dB}$ the maximum SNR loss of data transmissions in double-pulses, it benefits data transmissions in single-pulses significantly, reducing their maximum SNR loss by $0.69 \mathrm{~dB}$. Therefore, we emphasize the importance of optimizing the number of LDACS channels needed to cover a certain area, such that the same LDACS performance is achieved with the minimum number of LDACS frequency channels.

A variation in the LDACS cell radius around its average value of $120 \mathrm{nmi}$ might also affect the impact of LDACS on JTIDS. Thus, we repeat the simulations for both a lower cell radius $R_{\mathrm{L}}=60 \mathrm{nmi}$ with $E I R P_{\mathrm{GS}}=42 \mathrm{dBm}$ and a higher cell radius $R_{\mathrm{L}}=200 \mathrm{nmi}$ with $E I R P_{\mathrm{GS}}=52 \mathrm{dBm}$. In order to obtain a range of the SNR loss for each cell size, we consider the two locations of the $\mathrm{JU}_{\mathrm{Rx}}$ where practically the maximum and the minimum degradation were previously obtained. As it can be seen in the simulation results, shown jointly in Fig. 10 for data transmitted in STD and P2SP message structures, the impact of LDACS on JTIDS is maximum when the densest LDACS deployment is considered, despite its lower EIRP. The degradation suffered by data transmitted in double-pulses is lower than $0.35 \mathrm{~dB} \mathrm{~dB}$ for $R_{\mathrm{L}}=200 \mathrm{nmi}$ and $0.76 \mathrm{~dB}$ for $R_{\mathrm{L}}=60 \mathrm{nmi}$. When data are transmitted in single-pulses, the degradation of JTIDS for BER $=10^{-6}$ oscillates within the range $\Delta S N R \in[0.06,1.09] \mathrm{dB}$ for $R_{\mathrm{L}}=200 \mathrm{nmi}$ and $\Delta S N R \in[1.85,3.67] \mathrm{dB}$ for $R_{\mathrm{L}}=60$ nmi. Therefore, it can be seen that, even though doublepulse transmissions are not strongly affected by the considered LDACS cell size, single-pulse transmissions might suffer a significantly harmful LDACS interference when a very dense LDACS deployment is considered. However, this worst-case scenario can be avoided by following an appropriate LDACS cell planning. Specifically, if a dense LDACS deployment is required for some specific areas, LDACS cell planning should assign the lowest frequencies of both FL and RL bands to the cells covering those areas, in order to affect the minimum number of JTIDS frequency channels as possible.

In order to evaluate how a variation in the RL duty cycle might affect the impact of LDACS on JTIDS, we repeat the simulations for different values of $D$. Fig. 11 shows the simulation results for data transmitted in STD and P2SP message structures when the $\mathrm{JU}_{\mathrm{Rx}}$ is flying directly over a GS at an altitude of $18.288 \mathrm{~km}$. The SNR loss of the data transmitted in double-pulses increases from $0.38 \mathrm{~dB}$ for $D=0$, i.e., no interference from ASs, to $0.60 \mathrm{~dB}$ for $D=1$. Comparatively, the SNR loss of the data transmitted in single-pulses presents a slightly higher variation with the duty cycle, increasing from $1.64 \mathrm{~dB}$ for $D=0$, to $2.01 \mathrm{~dB}$ for $D=1$. Therefore, it is recommendable to prioritize the assignment of the lowest FL and RL LDACS frequencies to the cells which are expected to have the highest traffic loads in order to minimize their impact on JTIDS.

Up to now, only QPSK modulation has been considered for LDACS. However, as LDACS can use QPSK, 16-QAM, and 64-QAM modulation schemes adaptively in both FL and RL, we have also analysed how a different modulation scheme

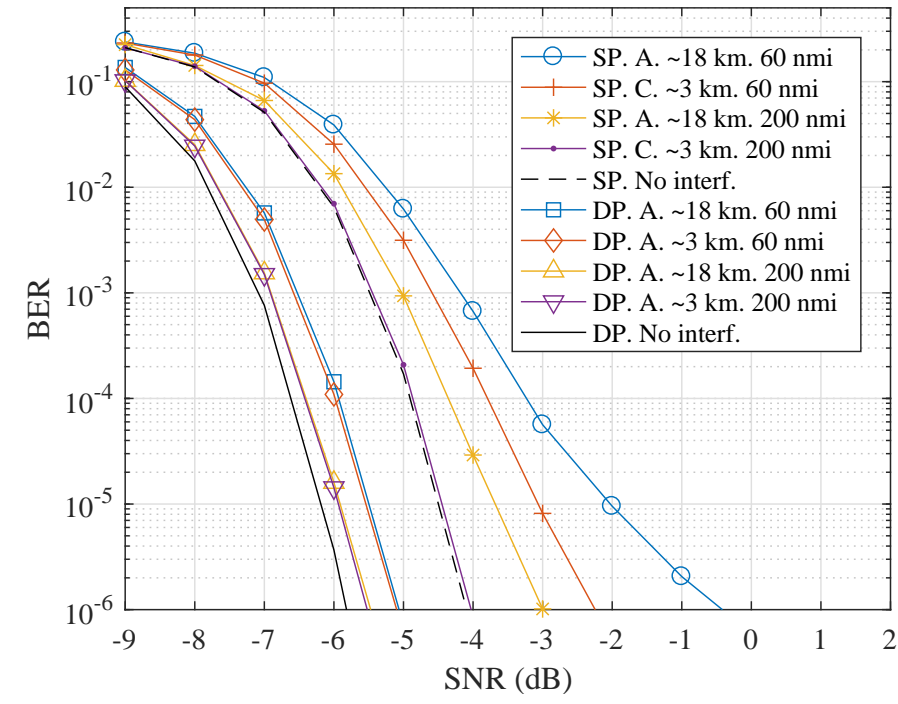

Fig. 10. Bit error rate of the data in P2SP (marked with "SP") and STD (marked with "DP") messages for different cell radii $R_{\mathrm{L}}$ and $E I R P_{\mathrm{GS}}$. Without LDACS interference ("No interf." curves) and with LDACS interference (other curves). $N_{\mathrm{f}}=12 . D=0.5$.

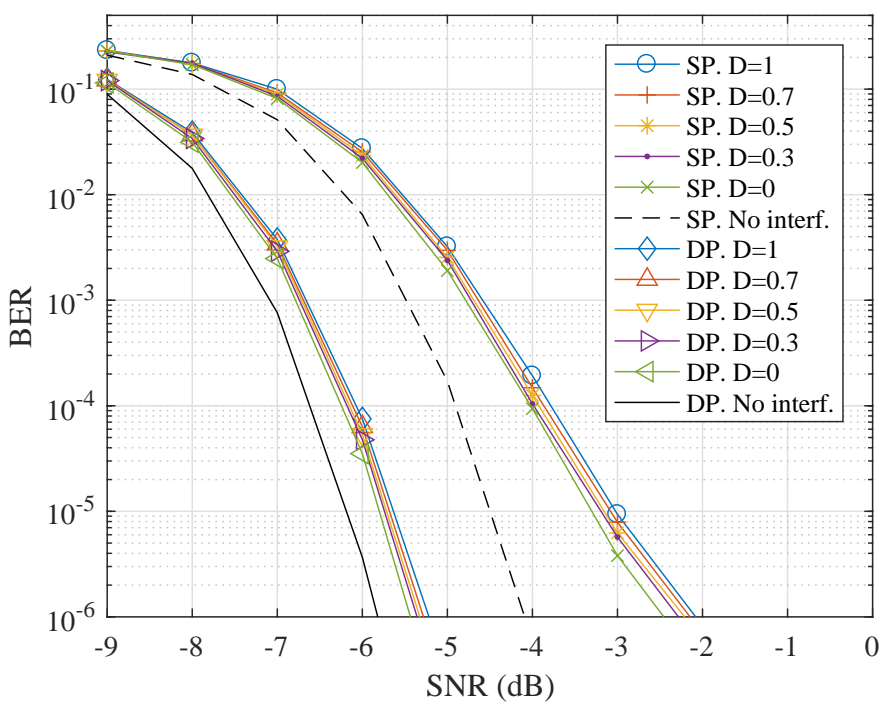

Fig. 11. Bit error rate of the data in P2SP (marked with "SP") and STD (marked with "DP") messages for different RL duty cycles $D$. Without LDACS interference ("No interf." curves) and with LDACS interference (other curves). $N_{\mathrm{f}}=12, R_{\mathrm{L}}=120 \mathrm{nmi}$. $E I R P_{\mathrm{GS}}=47 \mathrm{dBm}$.

might affect JTIDS transmissions. The simulation results, obtained for a JTIDS transmission of data in single-pulses when the $\mathrm{JU}_{\mathrm{Rx}}$ is flying over a GS at an altitude of 18.288 $\mathrm{km}$, show practically the same SNR loss independently on the modulation scheme used by LDACS. However, it is to be noted that the usage of a higher modulation scheme in the RL of a cell should reduce its duty cycle and, consequently, its impact on JTIDS.

\section{CONCLUSION}

In this paper, we analysed the impact that the future aeronautical communications system LDACS is expected to have on the military communications system JTIDS. The impact of LDACS on JTIDS has been quantified through 
simulations by comparing the performance of JTIDS obtained without LDACS interference, with the performance obtained under LDACS interference. Whilst the implemented models of LDACS transmitters are based on its current specification, the models of the JTIDS transmitter and receiver are based on publicly available information. For the simulation of the interference scenario between both systems, a wide variety of interference configurations has been considered.

Given that no official criteria are available to define under what interference conditions JTIDS will be harmfully affected, no assertion can be made from this perspective. However, simulation results show that JTIDS transmissions using double-pulses are effectively protected against LDACS interference due to the repetition of information at distant frequencies. In fact, the SNR loss of a JTIDS transmission in double-pulses remains below $1 \mathrm{~dB}$ for $\mathrm{BER}=10^{-6}$ in any considered case. In addition, the SNR required by the doublepulse transmissions to achieve a certain BER is, even for the strongest LDACS interference considered in our analysis, lower than the SNR required by single-pulse transmissions without any LDACS interference. Therefore, we can expect that the future deployment of LDACS will have a marginal impact on JTIDS transmissions using either the STD or the P2DP message structures. Comparatively, data transmissions in single-pulses are less protected against LDACS interference and a certain dependency on the specific LDACS deployment is observable. However, an appropriate LDACS cell planning following the recommendations given in our paper can restrict the SNR loss of data transmissions in single-pulses to less than $2 \mathrm{~dB}$ for $\mathrm{BER}=10^{-6}$, even under the conservative assumptions made in our analysis for a higher impact of LDACS on JTIDS.

\section{REFERENCES}

[1] International Civil Aviation Organization, "Air navigation report 2016 edition," 2016.

[2] STATFOR Team, "Eurocontrol seven-year forecast February 2017. Flight movements and service units 2017-2023," EUROCONTROL, 2017.

[3] B. Kamali, "An overview of VHF civil radio network and the resolution of spectrum depletion," in Proc. IEEE Integr. Commun. Navigat. Surveillance Conf., Herndon, VA, USA, May 2010, pp. F4-1-F4-8.

[4] Final acts WRC-07. International Telecommunication Union, 2007.

[5] SESAR homepage: http://www.sesarju.eu/.

[6] NextGen homepage: https://www.faa.gov/nextgen/.

[7] M. Sajatovic, B. Haindl, U. Epple, and T. Gräupl, "Updated LDACS1 system specification," SESAR Joint Undertaking Report EWA04-1-T2D1, 2011.

[8] N. Fistas, "L-DACS2 System Definition Proposal: Deliverable D2," EUROCONTROL, May 2009.

[9] M. Schnell, U. Epple, D. Shutin, and N. Schneckenburger, "LDACS: future aeronautical communications for air-traffic management," IEEE Communications Magazine, vol. 52, no. 5, pp. 104-110, May 2014.

[10] B. Haindl, J. Meser, M. Sajatovic, S. Mller, H. Arthaber, T. Faseth, and M. Zaisberger, "LDACS1 conformance and compatibility assessment," in Proc. IEEE/AIAA 33rd Digit. Avionics Syst. Conf., Colorado Springs, CO, USA, Oct. 2014, pp. 3B3-1-3B3-11.

[11] U. Epple and M. Schnell, "Overview of legacy systems in L-band and its influence on the future aeronautical communication system LDACS1," IEEE Aeros. and Elect. Syst. Mag., vol. 29, no. 2, pp. 31-37, Feb. 2014.

[12] M. Hirschbeck and J. B. Huber, "OFDM receiver structure for a subband-selective mitigation of time-variant interference," IEEE Trans. Veh. Technol., vol. 65, no. 10, pp. 8130-8144, Oct. 2016

[13] U. Epple and M. Schnell, "Advanced blanking nonlinearity for mitigating impulsive interference in ofdm systems," IEEE Trans. Veh. Technol., vol. 66 , no. 1 , pp. 146-158, Jan. 2017.
[14] Understanding voice and data link networking. Northrop Grumman, 2013.

[15] Chairman of the Joint Chiefs of Staff, "Link 16 spectrum deconfliction," U.S. Department of Defense, Instruction 6232.01E, 2012.

[16] R. S. Lokuta, "JTIDS electromagnetic compatibility in the 960-1215 MHz band," The MITRE Corporation, Bedford, 1992.

[17] J. Micallef, R. Womersley, S. Dunkley, and I. Casewell, "FCI technology investigations: $\mathrm{L}$ band compatibility criteria and interference scenarios study-Deliverables S1-S7: L-band interference scenarios," HELIOS, Hampshire, U.K., Tech. Rep. P1031D005, Aug. 2009.

[18] C.-H. Kao, "Performance analysis of a JTIDS/Link-16-type waveform transmitted over slow, flat Nakagami fading channels in the presence of narrowband interference," dissertation, Naval Postgraduate School, Monterey, California, 2008.

[19] A. De Martino, Introduction to modern EW systems. Artech House Publishers, 2012

[20] International Civil Aviation Organization, "LDACS spectrum assignment and frequency allocation update," Working Paper 6 presented by FREQUENTIS in the CP/IWG/PT-T/4, Jan. 2018.

[21] W. J. Wilson, "Applying layering principles to legacy systems: Link 16 as a case study," in Proc. IEEE Mil. Commun. Conf., vol. 1, McLean, VA, USA, Oct. 2001, pp. 526-531.

[22] S. Pasupathy, "Minimum shift keying: A spectrally efficient modulation," IEEE Communications Magazine, vol. 17, no. 4, pp. 14-22, 1979.

[23] Deutsches Zentrum für Luft- und Raumfahrt e.V. (DLR), "Proposed L-band interference scenarios," EUROCONTROL, Doc. Ref. CIEA15_EN511.10, Doc. Ident. D1, Issue 1.0, Feb. 2008.

[24] G. L. Stüber, Principles of Mobile Communication. Kluwer Academic Publishers, 2002.

[25] F. Hoffmann, U. Epple, M. Schnell, and U. C. Fiebig, "Feasibility of LDACS1 cell planning in european airspace," in Proc. IEEE/AIAA 31 st Digit. Avionics Syst. Conf., Williamsburg, VA, USA, Oct. 2012, pp. 5E1$1-5 \mathrm{E} 1-13$

[26] M. Mostafa, M. A. Bellido-Manganell, and T. Gräupl, "Feasibility of cell planning for the L-band digital aeronautical communications system under the constraint of secondary spectrum usage," IEEE Transactions on Vehicular Technology, vol. 67, no. 10, pp. 9721-9733, 2018.

[27] Final acts WRC-15. International Telecommunication Union, 2015.

[28] EUROCONTROL/FAA Future Communications Study Operational Concepts and Requirements Team, "Evaluation scenarios," 2007, ver. 1.0 .

[29] - "Communications operating concept and requirements for the future radio system (COCR), ver. 2," 2007.

[30] J. D. Parsons, The mobile radio propagation channel. John Wiley \& Sons, 2000 